\title{
The Character of Transport Caused by ExB Drift Turbulence
}

\author{
Bruce D. Scott \\ Max-Planck-IPP, EURATOM Association, 85748 Garching, Germany \\ Dec 2001
}

The basic character of diffusive transport in a magnetised plasma depends on what kind of transport is modelled. ExB turbulence under drift ordering has special characteristics: it is nearly incompressible, and it cannot lead to magnetic flux diffusion if it is electrostatic. The ExB velocity is also related to the Poynting energy flux. Under quasineutral dynamics, electric fields are not caused by transport of electric charge but by the requirement that the total current is divergence free. Consequences for well constructed computational transport models are discussed in the context of a general mean field analysis, which also yields several anomalous transfer mechanisms not normally considered by current models.

PACS numbers: 52.25.Fi 91.25.Cw 52.30.-q 52.40.Nk 


\section{Introduction - Transport as Turbulent Diffusion}

Transport of thermal energy and particles in magnetically confined, laboratory plasmas is well known to be anomalous: much larger than transport by collisional diffusion and appearing with different scaling characteristics $[1,2]$. Modelling of this transport is usually done in terms of the two fluid Braginskii equations [3], but by substituting either empirical or theoretical models for the diffusivity coefficients [4]. The transport process is assumed by the models to have the same character as the collisional diffusion process, including the prospect that Onsager symmetry should hold [5]. It is important to note that the transport process is assumed by such a model to have the same character as the collisional diffusion process. Many of the results of kinetic transport theory rest on the assumption that fluctuations in the thermodynamic state variables are small enough to be neglected in the energy budget and that they are randomly correlated with each other.

Small scale, low frequency drift turbulence involving eddies, waves, or vortices of the ExB velocity,

$$
\mathbf{v}_{E}=\frac{c}{B^{2}} \mathbf{B} \times \nabla \phi
$$

where at drift scales the perpendicular electric field is $\mathbf{E}_{\perp}=-\nabla \phi$, is a fundamentally different process from this. Rather than involving dissipation directly, the ExB eddies advect the background thermal gradient to produce disturbances in all the thermodynamic state variables. The disturbances are then carried with the flows for as long as the eddies last. Then, they are picked up by new eddies and carried further. This does have the nature of a diffusive process if the size of the eddies and their lifetime are small and short compared to the scales of the background, and the net time-averaged transport does indeed scale with the background gradients if the turbulence is local [6]. Moreover, although the dynamics of the turbulence is robustly nonlinear, the relative amplitudes of the disturbances are indeed

small because the plasma is magnetised; the relative amplitudes $e \widetilde{\phi} / T_{e}$ or $\widetilde{p}_{e} / p_{e}$, for the electrostatic potential and electron pressure for example, need only be so large as the ratio of the local drift scale, $\rho_{s}$, to the background gradient scale length, $L_{p}$, both defined by

$$
\rho_{s}^{2}=\frac{c^{2} M_{i} T_{e}}{e^{2} B^{2}} \quad L_{p}=\left|\nabla \log p_{e}\right|^{-1}
$$

in order to be so nonlinear. Typical measured values of these fluctuation amplitudes are of order one to ten percent, rising towards the edge of a confined plasma due to the fact that $L_{p}$ becomes much smaller than the nominal parallel length scale $q R$ (the field line pitch parameter times the major radius of a toroidal plasma) and the perpendicular drift dynamics is more able to compete with the dissipation incurred through the parallel 
electron dynamics [7]. Nevertheless, the strong nature of the coupling mechanisms between the state variables (especially $\widetilde{p}_{e}$ and $\widetilde{\phi}$ ) means that the basic properties of this turbulence are those of nonlinear drift waves [8,9], under drift ordering - the scale of the turbulence is short compared to that of the background gradients and the relative amplitude of the fluctuations in the thermodynamics quantities is small [10]. Drift wave turbulence involves dynamics on scales which are proportional but not necessarily equal to $\rho_{s}$ for space and $c_{s} / L_{p}$ for time, for plasmas which have both the local drift parameter, $\delta$, and the electron dynamical beta, $\beta_{e}$, as small parameters. These two parameters and the sound speed, $c_{s}$, are given by

$$
\delta=\frac{\rho_{s}}{L_{p}} \quad \beta_{e}=\frac{4 \pi p_{e}}{B^{2}}=\frac{c_{s}^{2}}{v_{A}^{2}} \quad c_{s}^{2}=\frac{T_{e}}{M_{i}}
$$

where $v_{A}$ is the Alfvén velocity. The presence of $T_{e}$ and $M_{i}$ reflects coupling between electron thermal dynamics and ion inertia. Even with finite ion temperature $T_{i} \neq T_{e}$, the coupling between the electron pressure and the electric field keeps $\rho_{s}$ and $c_{s} / L_{p}$ as the typical scaling parameters of the disturbances. This coupling and the fact that one of the dynamical state variables $(\widetilde{\phi})$ serves simultaneously as the potential for the electric field and the stream function for the (essentially incompressible) flow eddies are the two most important properties of drift wave turbulence. These properties and the result that the disturbances are not statistically independent of each other have consequences concerning the nature of the transport process. Our interest in this article is to explore how these properties differ from those of the kinetic diffusion process behind classical (and neoclassical) transport processes, and what implications this has for well constructed transport models.

\section{The Basic Character of Small Scale ExB Flow Transport}

There are a number of fundamental properties of ExB drift turbulence which suggest that the transport they cause should be qualitatively different from random thermal transport. First, if conventional drift ordering holds the velocity is nearly incompressible. If the magnetic field is straight then the divergence $\nabla \cdot \mathbf{v}_{E}$ vanishes entirely. In a toroidal device, the divergence carries the scale of the major radius:

$$
\nabla \cdot \mathbf{v}_{E}=\nabla \cdot \frac{c}{B^{2}} \mathbf{B} \times \nabla \phi \approx-\mathbf{v}_{E} \cdot \nabla \log B^{2} \sim R^{-1} \mathbf{v}_{E}
$$

Since in the confinement zone the pressure scale length, $L_{p}$ is much smaller, we have

$$
\mathbf{v}_{E} \cdot \nabla \tilde{p} \gg \widetilde{p} \nabla \cdot \mathbf{v}_{E} \quad \text { if } \quad L_{p} \ll R
$$


Here, we assume that the scale of a significant divergence of the transport flux is $L_{p}$ rather than the much smaller scale of ExB disturbances, because a locally divergence free state for the fluxes should be established on the relatively fast time scale of the turbulence, not the transport. By contrast, a diffusive flux of the form $n_{e} \mathbf{v}_{D}=-D \nabla n_{e}$ is always compressible, as it can be derived from a potential.

The second important characteristic is that the $\mathrm{ExB}$ velocity is also involved in the Poynting energy flux,

$$
\mathbf{v}_{E}=\frac{c}{B^{2}} \mathbf{E} \times \mathbf{B}=\frac{1}{\rho v_{A}^{2}} c \frac{\mathbf{E} \times \mathbf{B}}{4 \pi}
$$

with $\rho$ the mass density. Due to the close force balance for small scale disturbances in drift dynamics, there is a cancellation between the Poynting flux and one factor of $p \mathbf{v}_{E}$ in the total thermal energy flux. This has the consequence that alone among transport mechanisms, for ExB drift turbulence the advective part of the thermal energy flux appears with a factor of $3 / 2$, not $5 / 2$, times the temperature times the particle flux [11]. More specifically, its energetic coupling to the thermal reservoir is mediated through its small, quasistatic divergence, so that the pressure does little work on elemental volumes of the fluid.

A third characteristic of ExB turbulent transport also arises from the connection between the flow stream function and the electric field: anomalous energy exchange channels between the electron and ion thermal energies. The basic mechanism underlying drift waves is the coupling between electron pressure and electrostatic disturbances through the parallel current: $\widetilde{p}_{e} \leftrightarrow \widetilde{J}_{\|} \leftrightarrow \widetilde{\phi}$. The second step in this is the Alfvénic coupling $\widetilde{J}_{\|} \leftrightarrow \widetilde{\phi}$ which is present in MHD. The first step is the adiabatic coupling $\widetilde{p}_{e} \leftrightarrow \widetilde{J}_{\|}$, part of which is the adiabatic response in the Ohm's law, by which pressure disturbances launch shear Alfvén waves along the background magnetic field due to the force balance for electrons parallel to the field. This adiabatic coupling is responsible for a high degree of correlation between the electrostatic potential and electron pressure disturbances, and it provides the means by which the free energy in the background gradient is passed to the ExB turbulence $[6,9]$. It can only be addressed by a proper two fluid model which treats electron and ion dynamics separately but self consistently. Since the ExB drift kinetic energy is essentially in the ions, and the conservation of charge keeps the dynamics quasineutral, a parallel current divergence in the Alfvén dynamics is balanced by a polarisation current divergence in the ions, and this can do work on the ion fluid. Through the disturbances, electron thermal energy taken out of the background gradient can be deposited in the ions. 
A fourth characteristic of ExB flows is that they cannot cause magnetic flux diffusion unless there is a significant amount of reconnection of magnetic field lines in the presence of a current gradient (that is, the drive mechanism should be this current gradient, rather than the thermal gradients as for drift turbulence). The magnetic flux occurs through the inductive part of the electric field in the force balance for electrons, that is, $\partial A_{\|} / \partial t$. Neglecting dissipation and electron inertia the Ohm's law is

$$
\frac{1}{c} \frac{\partial A_{\|}}{\partial t}=\frac{\nabla_{\|} p_{e}}{n_{e} e}-\nabla_{\|} \phi
$$

In an average over a closed flux surface the right hand side vanishes unless there are appreciable magnetic disturbances. When the disturbances follow from drift dynamics the relative phase shifts are such that magnetic transport effects tend to cancel out of the flux surface averages. Only when the current gradient is available as an energy source do these processes lead to appreciable transport, even if the thermal transport is robustly anomalous. By contrast, random thermal diffusion transports magnetic flux by the same mechanism as for particles and energy, and the flux diffusivity, $\eta_{\|} c^{2} / 4 \pi$, is even larger than the fluid diffusivities, proportional to $D_{e}=\rho_{e}^{2} \nu_{e}$, by a factor of $\beta_{e}^{-1}$.

Two further considerations involve the development of large scale ExB flows within the flux surfaces (we assume that the equilibrium is quiescent enough that there is no bulk flow across flux surfaces beyond the existence of the turbulence). For dynamics at drift scales, the disturbances as well as the background are deeply quasineutral. Charge differences are neutralised on time and space scales which are effectively arbitrarily small, so that while there can be nonvanishing electric fields, there is no significant charge density. In this context it is inappropriate to speak of a radial electric field generated by the divergence of a radial current. Rather, we have the constraint that the total current is divergence free, so that various pieces of the current which might have a divergence are balanced by a divergence in the polarisation current of the ions. This is relatively well known in a general sense, but we will illustrate it here specifically for the problem of mean flow generation. If source or loss mechanisms for either of the charge species are considered, these should be thought of as a torque on the ExB vorticity rather than a charge generation[12].

Finally, anomalous transport in the ExB vorticity results fundamentally from anomalous viscosity, either the fluid Reynolds stress or some correction arising from gyroviscosity. Under flux surface and short time scale averaging, it is a matter of the total radial polarisation drift (linear plus nonlinear) necessarily vanishing. An anomalous resistivity cannot lead to anomalous transport of ExB flows or, equivalently, generation of a radial electric 
field. This is a direct consequence of the fact that resistivity is a momentum conserving friction between electrons and ions.

In the rest of this article, we address the general equations of transport by small scale drift turbulence via mean field theory, and then underscore the above points via analysis of these equations.

\section{Global Fluid Drift Equations and Energy Conservation}

The general derivation of fluid drift equations for large scale motion proceeds similarly to the local treatments more familiar from the turbulence studies $[8,9]$. The the difference is mainly that we must keep track of the variability of the coefficients which depend on densities or temperatures. The density is the most important of these. It carries the consequence that the ion polarisation drift must be retained in advection if exact energy conservation is to hold. This result follows from the fact that proper consideration of the electron force balance (the adiabatic response) disallows the use of the more familiar MHD ordering in the derivation of global equations $[13,14,15]$. What we must do is to generalise sufficiently to retain a consistent superset of both approaches.

We begin with a neutral, single-component plasma with ions of mass $M_{i}$ and charge state $Z$ and electrons of mass $m_{e}$. For this treatment we will neglect electron inertia but keep $m_{e}$ in combination with $\nu_{e}$ in the formulae for the dissipative fluxes. The densities satisfy $n_{e}=Z n_{i}$. We assume that the local drift parameter, $\delta$, and the electron dynamical beta, $\beta_{e}$, defined in the Introduction, are small. In contrast to the familiar situation with drift equation models, no assumption concerning flute mode ordering in the derivatives is made during the derivation of the equations themselves and their energy theorem concerning spatial scales, even for B. The more familiar assumption of $k_{\|} \ll k_{\perp}$, or for a toroidally confined plasma $L_{p} \ll R$, is reserved for evaluating the properties of the transport considering that the disturbances involved in the turbulence do indeed satisfy local drift ordering.

The drift approximation basically states that perpendicular compressional wave dynamics is arbitrarily fast compared to the physics of interest and is therefore neglected in favor of a quasistatically evolving force balance perpendicular to the magnetic field lines. We therefore have a quasistatic perpendicular electric field, with magnetic induction appearing only in the parallel component,

$$
\mathbf{E}_{\perp}=-\nabla_{\perp} \phi \quad E_{\|}=-\frac{1}{c} \frac{\partial A_{\|}}{\partial t}-\nabla_{\|} \phi
$$


Correspondingly, the total magnetic field, $\mathbf{B}_{t}$, is given by the background, plus the principal disturbance arising from $A_{\|}$, plus a small compressional piece which evolves quasistatically, plus an even smaller correction needed to ensure that $\nabla \cdot \mathbf{B}_{t}=0$,

$$
\mathbf{B}_{t}=\mathbf{B}-\frac{\mathbf{B}}{B} \times \nabla A_{\|}+\widetilde{B} \frac{\mathbf{B}}{B}-\nabla_{\perp} \chi_{B}
$$

respectively. The symbols $\mathbf{B}$ and $B$ give the equilibrium magnetic field and its magnitude, while $\mathbf{b}$ is reserved for the combination of $\mathbf{B}$ and the piece involving $A_{\|}$, given by

$$
\mathbf{B}_{\perp}=-\frac{\mathbf{B}}{B} \times \nabla A_{\|}
$$

so that in describing parallel components we use

$$
\mathbf{b}=\frac{1}{B}\left(\mathbf{B}+\mathbf{B}_{\perp}\right) \quad \nabla_{\|}=\mathbf{b} \cdot \nabla
$$

Perpendicular components are described by the background field only,

$$
\nabla_{\perp}=-\mathbf{B} \times \frac{\mathbf{B} \times \nabla}{B^{2}} \quad \nabla_{\perp}^{2}=\nabla \cdot \nabla_{\perp}
$$

so we must note that with these definitions we cannot write $\nabla=\nabla_{\perp}+\mathbf{b} \nabla_{\|}$, for example. The Ampere's law involving $A_{\|}$is written with $\nabla_{\perp}^{2}$,

$$
\nabla_{\perp}^{2} A_{\|}=-\frac{4 \pi}{c} J_{\|}
$$

The compressional part of the magnetic field, involving $\widetilde{B}$, is written for generality but not used in a $\beta_{e} \ll 1$ treatment (it can be used to evaluate the Poynting energy flux in the energy theorem by assuming that $\nabla_{\perp}^{2}$ of the sum $B^{2}+8 \pi p$ is zero). The divergence correction, involving $\chi_{B}$, is never used; it is written only to preserve $\nabla \cdot \mathbf{B}_{t}=0$, and in practice it is always negligibly small.

The quasistatic force balance allows us to write the drift velocities and heat fluxes perpendicular to $\mathbf{B}$ in terms of the state variables. For electrons the diamagnetic fluxes are

$$
\mathbf{v}_{*}=-\frac{1}{n_{e} e} \frac{c}{B^{2}} \mathbf{B} \times \nabla p_{e} \quad \mathbf{q}_{e \wedge}=-\frac{5}{2} \frac{p_{e}}{e} \frac{c}{B^{2}} \mathbf{B} \times \nabla T_{e}
$$

and for ions

$$
\mathbf{u}_{*}=\frac{1}{n_{i} Z e} \frac{c}{B^{2}} \mathbf{B} \times \nabla p_{i} \quad \mathbf{q}_{i \wedge}=\frac{5}{2} \frac{p_{i}}{Z e} \frac{c}{B^{2}} \mathbf{B} \times \nabla T_{i}
$$


The ExB drift for both species and the diamagnetic current are given by

$$
\mathbf{v}_{E}=\frac{c}{B^{2}} \mathbf{B} \times \nabla \phi \quad \mathbf{J}_{*}=n_{e} e\left(\mathbf{u}_{*}-\mathbf{v}_{*}\right)=\frac{c}{B^{2}} \mathbf{B} \times \nabla p
$$

with $p=p_{e}+p_{i}$. We form the lowest order perpendicular fluid velocities resulting from these drifts,

$$
\mathbf{u}_{\perp}=\mathbf{v}_{E}+\mathbf{u}_{*} \quad \mathbf{v}_{\perp}=\mathbf{v}_{E}+\mathbf{v}_{*}
$$

In the parallel dynamics the dependent variables are $u_{\|}$and $J_{\|}$, with $v_{\|}$defined as

$$
v_{\|}=u_{\|}-\frac{J_{\|}}{n_{e} e}
$$

The parallel current is actually an auxiliary variable obtained from $A_{\|}$through the drift form of Ampere's law, Eq. (13) above, with $\nabla^{2}$ replaced by $\nabla_{\perp}^{2}$.

For the ions we keep the polarisation drift, $\mathbf{u}_{p}$, which is required in the current balances and also in the contributions of the ion pressure to energy conservation. The polarisation drift is obtained by inserting the lowest order ion velocity, $\mathbf{u}_{\perp}+u_{\|} \mathbf{b}$, into the ion inertia terms (including all components of the stress tensor), and solving anew for the total ion velocity,

$$
\mathbf{u}=\mathbf{u}_{\perp}+u_{\|} \mathbf{b}+\mathbf{u}_{p}
$$

For the electrons we neglect polarisation along with $m_{e}$, so that their total velocity is

$$
\mathbf{v}=\mathbf{v}_{\perp}+v_{\|} \mathbf{b}
$$

noting that $v_{\|}$is given in Eq. (18). The polarisation drift is given by

$$
n_{e} e \mathbf{u}_{p}=n_{i} M_{i} \frac{c}{B^{2}} \mathbf{B} \times\left(\frac{\partial}{\partial t}+\mathbf{u} \cdot \nabla\right)\left[\frac{c}{B^{2}} \mathbf{B} \times\left(\nabla \phi+\frac{\nabla p_{i}}{n_{e} e}\right)\right]+\frac{c}{B^{2}} \mathbf{B} \times\left(\nabla \cdot \boldsymbol{\Pi}_{*}\right)
$$

where we keep the gyroviscosity explicitly instead of just using the part which cancels $\mathbf{u}_{*} \cdot \nabla$, in order to preserve its symmetry. We can express the polarisation drift in terms of a potential,

$$
\mathbf{u}_{p}=-\frac{M_{i} c^{2}}{Z e B^{2}} \nabla_{\perp} \chi=\frac{M_{i}}{Z e} \frac{c}{B^{2}} \mathbf{B} \times\left(\frac{c}{B^{2}} \mathbf{B} \times \nabla \chi\right)
$$

following from the fact that it is primarily proportional to gradients of scalars. In contrast to the local treatments, the polarisation drift does indeed appear explicitly in the inertia, but only in the advective derivative. On the other hand, the inertial velocity itself is the lowest order version, whose perpendicular component is $\mathbf{u}_{\perp}$. Consequently, we construct the components of $\boldsymbol{\Pi}_{*}$ using only the lowest order velocities, $\mathbf{u}_{\perp}+u_{\|} \mathbf{b}$, as we will need to 
use the fact that $\boldsymbol{\Pi}_{*}: \nabla \mathbf{u}$ vanishes, for whichever vector $\mathbf{u}$ is used to construct $\boldsymbol{\Pi}_{*}$ (if this is done in detail it must be extended to include $\mathbf{q}_{i \wedge}$ in $\boldsymbol{\Pi}_{*}$ to get the gyroviscous cancellation [13] to work properly when the temperature and density gradients are equally steep [16]). We note here that everything having to do with ion inertia in the perpendicular force balance is encompassed within the polarisation drift, including not only the advection of vorticity but also Reynolds stress and gyroviscosity.

The equations under the drift approximation are formed by substituting the above expressions for the velocities into the rest of the fluid equations. The evolution of $\phi$ is controlled by the polarisation equation, which is formed by subtracting the densities so as to form a continuity equation for electric charge. The charge density itself is set to zero, leaving $\nabla \cdot \mathbf{J}=0$. Expressing the total current as

$$
\mathbf{J}=J_{\|} \mathbf{b}+\mathbf{J}_{*}+\mathbf{J}_{p}
$$

with the polarisation current given by $\mathbf{J}_{p}=n_{e} e \mathbf{u}_{p}$, we find

$$
-\nabla \cdot n_{e} e \mathbf{u}_{p}=\nabla \cdot\left(J_{\|} \mathbf{b}\right)+\nabla \cdot \frac{c}{B^{2}} \mathbf{B} \times \nabla\left(p_{e}+p_{i}\right)
$$

Where we need to evaluate $\mathbf{u}_{p}$ in the ion equations, we may use,

$$
\nabla \cdot \frac{n_{i} M_{i} c^{2}}{B^{2}} \nabla_{\perp \chi}=\nabla \cdot\left(J_{\|} \mathbf{b}\right)+\nabla \cdot \frac{c}{B^{2}} \mathbf{B} \times \nabla\left(p_{e}+p_{i}\right)
$$

which we solve for $\chi$. The evolution of $A_{\|}$and $J_{\|}$is controlled by the Ohm's law, which is just the parallel electron force balance including dissipation. The rest of the dependent variables in the set $\left\{\phi, n_{e}, A_{\|}, p_{e}, u_{\|}, p_{i}\right\}$ have their own fluid equations, in which we use the same procedure as in the polarisation equation. The resulting model is given by

$$
\begin{gathered}
-\nabla \cdot\left\{n_{i} M_{i} \frac{c}{B^{2}} \mathbf{B} \times\left(\frac{\partial}{\partial t}+\mathbf{u} \cdot \nabla\right)\left[\frac{c}{B^{2}} \mathbf{B} \times\left(\nabla \phi+\frac{\nabla p_{i}}{n_{e} e}\right)\right]+\frac{c}{B^{2}} \mathbf{B} \times\left(\nabla \cdot \mathbf{\Pi}_{*}\right)\right\}= \\
=\nabla \cdot\left(J_{\|} \mathbf{b}\right)+\nabla \cdot \frac{c}{B^{2}} \mathbf{B} \times \nabla\left(p_{e}+p_{i}\right) \\
\frac{\partial n_{e}}{\partial t}+\nabla \cdot\left[n_{e} \mathbf{v}_{E}+n_{e}\left(u_{\|}-\frac{J_{\|}}{n_{e} e}\right) \mathbf{b}-\frac{c}{B^{2}} \mathbf{B} \times \nabla \frac{p_{e}}{e}\right]=0 \\
\frac{1}{c} \frac{\partial A_{\|}}{\partial t}=\frac{\nabla_{\|} p_{e}}{n_{e} e}-\nabla_{\|} \phi-R_{e i} \\
\frac{\partial}{\partial t} \frac{3}{2} p_{e}+\nabla \cdot\left[\frac{3}{2} p_{e} \mathbf{v}_{E}+\frac{5}{2} p_{e}\left(u_{\|}-\frac{J_{\|}}{n_{e} e}\right) \mathbf{b}+q_{e} \mathbf{b}-\frac{c}{B^{2}} \mathbf{B} \times \nabla \frac{5}{2} \frac{p_{e} T_{e}}{e}\right]=
\end{gathered}
$$




$$
\begin{array}{r}
=u_{\|} \nabla_{\|} p_{e}-\frac{J_{\|}}{n_{e} e} \nabla_{\|} p_{e}-p_{e} \nabla \cdot \mathbf{v}_{E}+J_{\|} R_{e i} \\
n_{i} M_{i}\left(\frac{\partial}{\partial t}+\mathbf{u} \cdot \nabla\right) u_{\|}+\mathbf{b} \cdot\left(\nabla \cdot \mathbf{\Pi}_{*}\right)=-\nabla_{\|}\left(p_{e}+p_{i}\right)+\nabla \cdot\left(\mu_{\|} \mathbf{b} \nabla_{\|} u_{\|}\right) \\
\frac{\partial}{\partial t} \frac{3}{2} p_{i}+\nabla \cdot\left[\frac{3}{2} p_{i} \mathbf{v}_{E}+\frac{5}{2} p_{i} u_{\|} \mathbf{b}+q_{i \|} \mathbf{b}+\frac{5}{2} p_{i} \mathbf{u}_{p}+\frac{c}{B^{2}} \mathbf{B} \times \nabla \frac{5}{2} \frac{p_{i} T_{i}}{e}\right]= \\
=u_{\|} \nabla_{\|} p_{i}+\mathbf{u}_{p} \cdot \nabla p_{i}-p_{i} \nabla \cdot \mathbf{v}_{E}+\mu_{\|}\left|\nabla_{\|} u_{\|}\right|^{2}
\end{array}
$$

where except for $\nabla \cdot \mathbf{v}_{E}$ the magnetic divergences are written explicitly. The dissipative momentum transfer,

$$
R_{e i}=\eta_{\|}\left[J_{\|}+\frac{\alpha}{\kappa_{e}}\left(\alpha J_{\|}+\frac{e}{T_{e}} q_{e \|}\right)\right]
$$

and parallel heat fluxes,

$$
q_{e \|}+\alpha \frac{T_{e}}{e} J_{\|}=-\kappa_{e} n_{e} \frac{V_{e}^{2}}{\nu_{e}} \nabla_{\|} T_{e} \quad q_{i \|}=-\kappa_{i} n_{i} \frac{V_{i}^{2}}{\nu_{i}} \nabla_{\|} T_{i}
$$

are given by the Braginskii model, with the resistivity and electron and ion thermal velocities given by

$$
\eta_{\|}=\eta \frac{m_{e} \nu_{e}}{n_{e} e^{2}} \quad V_{e}=\left(\frac{T_{e}}{m_{e}}\right)^{1 / 2} \quad V_{i}=\left(\frac{T_{i}}{M_{i}}\right)^{1 / 2}
$$

respectively, with numerical constants for $Z=1$

$$
\eta=0.51 \quad \alpha=0.71 \quad \kappa_{e}=3.2 \quad \kappa_{i}=3.9
$$

and the collision frequencies given by the inverses of the Braginskii collision times [3].

We note that this is an approximate treatment, but as we will now show, it conserves global energy. We first make a few remarks. First, the reason for including $\mathbf{u}_{p}$ in the advective derivative $\mathbf{u} \cdot \nabla$ is conservation of the inertial energy incorporated in $\mathbf{u}_{\perp}$. We must manipulate the polarisation equation such that the density is brought under both the time derivative and under a resulting total divergence. Since it starts out as a multiplier on $\partial / \partial t$ as well as on the advecting velocity, the continuity demands require that we keep $\mathbf{u}_{p}$ in $n_{i} \mathbf{u} \cdot \nabla$ if we keep it in $\nabla \cdot n_{i} \mathbf{u}$. This chain of demands starts at the presence of $\nabla_{\|} p_{e}$ in the Ohm's law, continues with $\nabla_{\|} J_{\|}$in the electron pressure equation and hence the density equation, and finally with the fact that the divergences of $J_{\|}$and $\mathbf{u}_{p}$ go together in the continuity. The ordering and conservation are preserved by keeping $\mathbf{u}_{p}$ in $\mathbf{u} \cdot \nabla$, but not in the actual inertial velocity, $\mathbf{u}_{\perp}$. Second, although the perpendicular and parallel 
components of vectors are set up differently, they are kept separate in all computations so that there is no violation of energy conservation. Finally, one sees that part of the drift ordering is expressed in the fact that the equations are written only for the lowest order components of the ion velocity and magnetic field, but the operations involving those velocities and gradients include all components in order to conserve energy.

The energy theorem is formed by multiplying Eqs. $(26,30)$ for the vorticity and parallel velocity by $-\phi$ and $u_{\|}$, and Eq. (28) for the magnetic potential by $J_{\|}$, respectively. The pressures in Eqs. $(29,31)$ represent the thermal energy. The total energy density is

$$
U=\int d^{3} x\left(n_{i} M_{i} \frac{\left|\mathbf{u}_{\perp}\right|^{2}}{2}+n_{i} M_{i} \frac{u_{\|}^{2}}{2}+\frac{3}{2} p_{e}+\frac{3}{2} p_{i}+\frac{\left|\mathbf{B}_{\perp}\right|^{2}}{8 \pi}\right)
$$

We will call these pieces the drift energy, sound wave energy, electron and ion thermal energy, and magnetic energy, respectively.

The first consideration is how polarisation works under the drift approximation. We multiply the polarisation equation by $\phi$, to obtain

$$
\phi \nabla \cdot \mathbf{J}=\nabla \cdot \phi \mathbf{J}-\mathbf{J} \cdot \nabla \phi=0
$$

where $\mathbf{J}$ includes all three pieces, $J_{\|} \mathbf{b}+\mathbf{J}_{*}+\mathbf{J}_{p}$. The form of this that we actually use is

$$
-\mathbf{J}_{p} \cdot \nabla \phi+\nabla \cdot \phi \mathbf{J}-\mathbf{J}_{*} \cdot \nabla \phi=J_{\|} \nabla_{\|} \phi
$$

where the first piece will give part of the drift energy, the next two pieces will be combined into a total flux plus a transfer term, and the piece on the right side is already a transfer term. We manipulate

$$
\mathbf{J}_{*} \cdot \nabla \phi=\left(\frac{c}{B^{2}} \mathbf{B} \times \nabla p\right) \cdot \nabla \phi=-\left(\frac{c}{B^{2}} \mathbf{B} \times \nabla \phi\right) \cdot \nabla p=-\mathbf{v}_{E} \cdot \nabla p
$$

where $p=p_{e}+p_{i}$ is the total pressure, and then combine with the total divergence to write

$$
-\mathbf{J}_{p} \cdot \nabla \phi+\nabla \cdot\left(\phi \mathbf{J}+p \mathbf{v}_{E}\right)=J_{\|} \nabla_{\|} \phi+p \nabla \cdot \mathbf{v}_{E}
$$

We note that in general the divergence of $\phi \mathbf{J}$ is equivalent to part of the Poynting flux,

$$
\nabla \cdot \phi \mathbf{J}=\nabla \cdot\left(\frac{c}{4 \pi} \phi \nabla \times \mathbf{B}_{t}\right)=-\nabla \cdot\left(\frac{c}{4 \pi} \nabla \phi \times \mathbf{B}_{t}\right)
$$

dropping a divergence of a curl. The near cancellation with $p \mathbf{v}_{E}$ is called the Poynting cancellation,

$$
\nabla \cdot\left(\phi \mathbf{J}_{\perp}+p \mathbf{v}_{E}\right) \approx 0
$$


affecting $\mathbf{J}_{\perp}$ since $\mathbf{v}_{E}$ is also perpendicular to $\mathbf{B}$. This cancellation results in the fact that the ExB thermal transport eventually appears with a factor of $3 / 2$, so that the ExB divergence, not an ExB advection, is the process by which transfer with the thermal reservoir occurs. We will show this in Section $\mathrm{V}$, together with the other characteristics of ExB transport.

The part of the Poynting flux involving $J_{\|}$moves parallel to $\mathbf{B}$ and is the process by which the MHD part of shear Alfvén waves move field energy along B:

$$
\nabla \cdot\left(\phi J_{\|} \mathbf{b}\right) \approx-\nabla \cdot\left(\frac{c}{4 \pi} \nabla \phi \times \mathbf{B}_{\perp}\right)
$$

neglecting the action of the curl on $\mathbf{b}$ itself. This is generally comparable to or larger than $\nabla \cdot\left(p_{e} v_{\|} \mathbf{b}\right)$, reflecting the properties of shear Alfvén dynamics under general two fluid ordering.

The pressure piece in the drift energy is found by subtracting $\mathbf{u}_{p} \cdot \nabla p_{i}$ from both sides of Eq. (40), to find

$$
-\mathbf{J}_{p} \cdot\left(\nabla \phi+\frac{\nabla p_{i}}{n_{e} e}\right)+\nabla \cdot\left(\phi \mathbf{J}+p \mathbf{v}_{E}\right)=J_{\|} \nabla_{\|} \phi+\left(p_{e}+p_{i}\right) \nabla \cdot \mathbf{v}_{E}-\mathbf{u}_{p} \cdot \nabla p_{i}
$$

Finally, substituting in for $\mathbf{J}_{p}$ and noting how the combination in parentheses gives rise to $\mathbf{u}_{\perp}$, we have

$$
\begin{aligned}
\frac{\partial}{\partial t}\left(n_{i} M_{i} \frac{\left|\mathbf{u}_{\perp}\right|^{2}}{2}\right) & +\nabla \cdot\left[n_{i} M_{i} \frac{\left|\mathbf{u}_{\perp}\right|^{2}}{2} \mathbf{u}+\mathbf{u}_{\perp} \cdot \boldsymbol{\Pi}_{*}+\phi \mathbf{J}+p \mathbf{v}_{E}\right]= \\
& =\boldsymbol{\Pi}_{*}: \nabla \mathbf{u}_{\perp}+J_{\|} \nabla_{\|} \phi+\left(p_{e}+p_{i}\right) \nabla \cdot \mathbf{v}_{E}-\mathbf{u}_{p} \cdot \nabla p_{i}
\end{aligned}
$$

in which the transport terms are the ones under the divergence operator on the left side, and the transfer terms are those on the right side. We note that the reason that $\mathbf{u}$ must include all components of the ion velocity is to bring the factor of $n_{i}$ under both the partial time derivative and the divergence operator simultaneously. In other words, whatever we keep in the velocity divergence in the density equation must also be kept in the advection in the polarisation equation, and in all the ion fluid equations as well.

We now find where the energy transferred out of this drift energy goes. The next subtlety is the magnetic energy. We multiply Eq. (28) by $J_{\|}$and manipulate the divergence to obtain

$$
\frac{\partial}{\partial t} \frac{1}{8 \pi}\left|\nabla_{\perp} A_{\|}\right|^{2}+\nabla \cdot\left(\nabla_{\perp} A_{\|} \frac{1}{4 \pi c} \frac{\partial A_{\|}}{\partial t}\right)=\frac{J_{\|}}{n_{e} e} \nabla_{\|} p_{e}-J_{\|} \nabla_{\|} \phi-J_{\|} R_{e i}
$$


The terms on the right side are the adiabatic and Alfvénic transfer effects and resistive dissipation, which function the same way as in drift wave turbulence [6]. The divergence term is the inductive part of the Poynting energy flux, as we can see by evaluating the projection operations implicit in the $\nabla_{\perp}$ operator,

$$
\nabla \cdot\left(\nabla_{\perp} A_{\|} \frac{1}{4 \pi c} \frac{\partial A_{\|}}{\partial t}\right)=\nabla \cdot \frac{c}{4 \pi}\left[\frac{1}{c} \frac{\mathbf{B}}{B} \frac{\partial A_{\|}}{\partial t} \times\left(\frac{\mathbf{B}}{B} \times \nabla A_{\|}\right)\right]
$$

Substituting $\mathbf{B}_{\perp}$ for $A_{\|}$, this gives the conservation law for the perturbed magnetic energy

$$
\frac{\partial}{\partial t} \frac{\left|\mathbf{B}_{\perp}\right|^{2}}{8 \pi}-\nabla \cdot \frac{c}{4 \pi}\left(\frac{1}{c} \mathbf{b} \frac{\partial A_{\|}}{\partial t} \times \mathbf{B}_{\perp}\right)=\frac{J_{\|}}{n_{e} e} \nabla_{\|} p_{e}-J_{\|} \nabla_{\|} \phi-J_{\|} R_{e i}
$$

where we note that the inductive electric field appears with $\mathbf{b}$ since the term it is crossed into is $\mathbf{B}_{\perp}$. The inductive part of the Poynting flux involving $\mathbf{B}_{\perp}$ is in this equation, transporting magnetic energy, while the static part is in Eq. (43), transporting ExB energy. Both are small, however, except for the part propagating along $\mathbf{B}$, which carries the Alfvén wave energy but whose contribution to transport across magnetic flux surfaces is nevertheless negligible.

The sound wave energy is found by multiplying Eq. (30) by $u_{\|}$, noting that here also the need to put the factor of $n_{i}$ under the time derivative and the divergence requires keeping $\mathbf{u}_{p}$ in the advection terms. We obtain

$$
\begin{aligned}
\frac{\partial}{\partial t}\left(n_{i} M_{i} \frac{u_{\|}^{2}}{2}\right)+\nabla \cdot\left(n_{i} M_{i} \frac{u_{\|}^{2}}{2} \mathbf{u}+u_{\|} \mathbf{b} \cdot \mathbf{\Pi}_{*}-u_{\|} \mathbf{b} \mu_{\|} \nabla_{\|} u_{\|}\right)= \\
=\boldsymbol{\Pi}_{*}: \nabla\left(u_{\|} \mathbf{b}\right)-u_{\|} \nabla_{\|}\left(p_{e}+p_{i}\right)-\mu_{\|}\left|\nabla_{\|} u_{\|}\right|^{2}
\end{aligned}
$$

noting that the gyroviscosity term cancels properly with the one in Eq. (45) because $\boldsymbol{\Pi}_{*}: \nabla\left(\mathbf{u}_{\perp}+u_{\|} \mathbf{b}\right)$ vanishes. Gyroviscosity thereby represents a diamagnetic momentum flux which acts to transfer energy between parallel and perpendicular fluid motion.

The thermal energy comes next. These are simply the pressure equations, with some of the advection terms evaluated piece by piece. For the electrons we have Eq. (29), in which the transfer effects with other equations are now obvious: adiabatic transfer $\left(J_{\|} \nabla_{\|} p_{e}\right)$ and resistive frictional heating $\left(J_{\|} R_{e i}\right)$ with the magnetic energy, acoustic coupling $\left(u_{\|} \nabla_{\|} p_{e}\right)$ with the sound waves, and the ExB divergence $\left(p_{e} \nabla \cdot \mathbf{v}_{E}\right)$ with the drift energy through polarisation. For the ions we have Eq. (31), in which the transfer effects are thermal coupling $\left(u_{\|} \nabla_{\|} p_{i}\right)$ and viscous heating (the $\mu_{\|}$term) with the sound waves, and the ExB 
divergence $\left(p_{i} \nabla \cdot \mathbf{v}_{E}\right)$ through polarisation and the polarisation advection $\left(\mathbf{u}_{p} \cdot \nabla p_{i}\right)$ with the drift energy.

The total energy theorem is then given by

$$
\begin{aligned}
& \frac{\partial U}{\partial t}+\nabla \cdot\left[\frac{3}{2}\left(p_{e}+p_{i}\right) \mathbf{v}_{E}+\left(q_{e \|}+q_{i \|}\right) \mathbf{b}+\right. \\
& \quad+\frac{5}{2}\left(\phi J_{\|}+p_{e} v_{\|}+p_{i} u_{\|}\right) \mathbf{b}+\frac{5}{2} p_{i} \mathbf{u}_{p}+\frac{c}{B^{2}} \mathbf{B} \times \nabla \frac{5}{2} \frac{p_{i} T_{i}}{e}-\frac{c}{B^{2}} \mathbf{B} \times \nabla \frac{5}{2} \frac{p_{e} T_{e}}{e}+ \\
& \left.+n_{i} M_{i} \frac{\left|\mathbf{u}_{\perp}\right|^{2}+u_{\|}^{2}}{2} \mathbf{u}+\mathbf{\Pi}_{*} \cdot\left(\mathbf{u}_{\perp}+u_{\|} \mathbf{b}\right)+\left(\phi \mathbf{J}_{\perp}+p \mathbf{v}_{E}\right)-\frac{c}{4 \pi} \frac{1}{c} \mathbf{b} \frac{\partial A_{\|}}{\partial t} \times \mathbf{B} \perp\right]=0
\end{aligned}
$$

The transport effects are written in order of their usual importance; in practical situations only the terms in the first line need be kept. In situations where transport by Pfirsch-Schlüter currents (generally caused by nonzero magnetic divergences involving the equilibrium pressure) is relevant, the second line would have to be kept as a unit, since the divergences of all the currents go together. The diamagnetic fluxes are combined into the terms involving the drift operator. The terms in the last line are negligible unless there are transonic or supersonic flows.

The dominant transport effect across the magnetic flux surfaces is the ExB advection, $(3 / 2) p_{e} \mathbf{v}_{E}$, which appears with the factor of $3 / 2$ due to the Poynting cancellation, Eq. (42). The diamagnetic fluxes drop to the second line due to the well known diamagnetic cancellation in the pressure equations [17]. For the parallel transport, the principal effects are the electron and ion heat fluxes, $B^{-1}\left(q_{e \|}+q_{i \|}\right) \mathbf{B}_{\perp}$. We also have the ion advection, $(5 / 2 B) p_{i} u_{\|} \mathbf{B}_{\perp}$, appearing with the factor of $5 / 2$ due to the compressibility of the parallel flows. For the electrons the part of this effect involving $J_{\|}$is small because $A_{\|}$is the stream function for $\mathbf{B}_{\perp}$ and it is also related to $J_{\|}$through Ampere's law, but here we also have the acoustic advective effect, $(5 / 2 B) p_{e} u_{\|} \mathbf{B}_{\perp}$. These parallel advective effects are small, however, since the sound wave transit time is generally much longer than the time scale of the turbulence.

To summarise, we have a set of drift equations following the drift ordering as to magnetic compression but allowing for an arbitrary scale of motion and with it, interactions with the thermal gradient of the background. The conservation of total energy is exact, but at the price that the total velocity must be kept in advection. The extra piece, that is, the polarisation drift, is given in terms of the dependent variables through a constitutive relation, Eq. (25). 


\section{A One Dimensional Mean Field Model of Transport by ExB Turbulence}

In this section we construct a mean field transport model including both perpendicular and parallel flows. All dependent variables are split into profile quantities which are flux functions (dependence only on the flux surface label coordinate), and disturbances which have arbitrary coordinate dependence but are zero in ensemble average. The profiles are assumed to be slowly varying in time and space compared to the disturbances, so the ensemble average can be understood as one over an interval of time short compared to the transport time of a given radial region but long compared to the fluctuation time scale of the turbulence. The energy content of the disturbances is assumed to be negligible, so that their energy equations become statements that their net transfer effects are all in quasistatic balance. Transport of wave energy across flux surfaces (disturbance energy content times $\widetilde{\mathbf{v}}_{E}$ ) is kept until the disturbance energy equations are evaluated, in order to show that it is small. The disturbances form nonvanishing quadratic transport and transfer quantities in the mean field equations; these quantities control the evolution of the profiles.

We retain both poloidal and toroidal rotation, fully described by the two state variables, $\phi$ and $p_{i}$, and the parallel flow $u_{\|}$. In contrast with more usual treatments, we keep the partial time derivatives in the polarisation equation, and hence also the energy content of the drift flows. Both flows are affected by Reynolds stress and by neoclassical friction, the latter acting only on the poloidal component of the total flow (recall that parallel is not purely toroidal and perpendicular is not purely poloidal). The parallel flow disturbances are also dissipated by parallel viscosity. We also retain magnetic flux diffusion, keeping resistive dissipation for both profiles and disturbances.

The equations for the profiles are those derived in Section III, simplified by the symmetry assumptions, but with the addition of the ensemble averaged transport effects. For the disturbances we mainly have to declare what is kept in order to satisfy energy conservation, since the role of the disturbances is to provide transfer channels.

To proceed with the transfer evaluations we need the density equation. This model follows transport of electrons, but the ion density is needed for the factors of $n_{i} M_{i}$ in front of the partial time derivatives in the flow equations to conserve energy properly. For the electrons we have

$$
\frac{\partial n_{e}}{\partial t}+\nabla \cdot\left\langle\widetilde{n}_{e} \widetilde{\mathbf{v}}_{E}\right\rangle=0
$$


neglecting compression effects. With this simplification, we have equivalently the same equation for the ions

$$
\frac{\partial n_{i}}{\partial t}+\nabla \cdot\left\langle\widetilde{n}_{i} \widetilde{\mathbf{v}}_{E}\right\rangle=0
$$

We write both these equations to emphasise the fact that neglect of the polarisation compression and the neglect of particle transport by the parallel current in general go together. It is important here to note the assumption that $\nabla \cdot n_{i} \widetilde{\mathbf{v}}_{E}$ can be neglected, since the density-weighted average of $\widetilde{\mathbf{v}}_{E}$ should vanish in order to have transport not go by direct flow but by statistical average. This helps in the manipulation of the Reynolds stresses.

The parallel flow profile equation is

$$
n_{i} M_{i} \frac{\partial u_{\|}}{\partial t}+M_{i}\left\langle\widetilde{n}_{i} \widetilde{\mathbf{v}}_{E}\right\rangle \cdot \nabla u_{\|}+\nabla \cdot n_{i} M_{i}\left\langle\widetilde{u}_{\|} \widetilde{\mathbf{v}}_{E}\right\rangle+n_{i} M_{i} \nu_{d} u^{\theta} b^{\theta}=0
$$

in which we have used the ability to neglect $\nabla \cdot n_{i} \widetilde{\mathbf{v}}_{E}$ before taking the ensemble average. The last term is the parallel component of the poloidal friction, with $u^{\theta}=\left(\mathbf{u}_{\perp}+u_{\|} \mathbf{b}\right) \cdot \nabla \theta$ the total poloidal flow component, $b^{\theta}=\mathbf{b} \cdot \nabla \theta$ the poloidal component of the magnetic unit vector, $\nu_{d}$ the neoclassical friction coefficient, and $\mathbf{u}_{\perp}$ given by the profiles of $\phi$ and $p_{i}$ as in Section III. The third term is the "conductive" parallel momentum transport, as we can see by using Eq. (52) to form the parallel momentum equation,

$$
\frac{\partial}{\partial t}\left(n_{i} M_{i} u_{\|}\right)+\nabla \cdot\left(M_{i}\left\langle\widetilde{n}_{i} \widetilde{\mathbf{v}}_{E}\right\rangle u_{\|}+n_{i} M_{i}\left\langle\widetilde{u}_{\|} \widetilde{\mathbf{v}}_{E}\right\rangle\right)+n_{i} M_{i} \nu_{d} u^{\theta} b^{\theta}=0
$$

The first term under the divergence is transport via the anomalous particle flux, and the second is due to correlations between the parallel flow and ExB disturbances, which is also the perp-parallel Reynolds stress. The latter term is called the "conductive" part of the transport. The transport operating through the particle flux is called the "convective" part. We simply generalise this concept, which is standard for thermal energy, to the other conserved quantities. The last term transfers momentum with the magnetic field; the momentum of $\mathbf{u}_{\perp}$ is not followed, consistent with the drift approximation in which the background magnetic field is an anchor.

For the parallel flow disturbances we have

$$
n_{i} M_{i} \frac{\partial \widetilde{u}_{\|}}{\partial t}+n_{i} M_{i} \widetilde{\mathbf{v}}_{E} \cdot \nabla \widetilde{u}_{\|}+M_{i}\left\langle\widetilde{n}_{i} \widetilde{\mathbf{v}}_{E}\right\rangle \cdot \nabla \widetilde{u}_{\|}+n_{i} M_{i} \widetilde{\mathbf{v}}_{E} \cdot \nabla u_{\|}=-\nabla \widetilde{p}+\mu_{\|} \nabla_{\|}^{2} \widetilde{u}_{\|}
$$

where $\widetilde{p}=\widetilde{p}_{e}+\widetilde{p}_{i}$ is the total pressure disturbance. The second and third terms are nonlinear advection, which is required in light of Eq. (52) by the need to get the factor of $n_{i} M_{i}$ under the time derivative. The fourth term is the drive term arising from the 
background flow gradient. It conserves energy against the perp-parallel Reynolds stress in the profile equation. The energy equations are given by

$$
\begin{array}{r}
\frac{\partial}{\partial t}\left(n_{i} M_{i} \frac{u_{\|}^{2}}{2}\right)+\nabla \cdot\left(M_{i}\left\langle\widetilde{n}_{i} \widetilde{\mathbf{v}}_{E}\right\rangle \frac{u_{\|}^{2}}{2}+n_{i} M_{i}\left\langle\widetilde{u}_{\|} \widetilde{\mathbf{v}}_{E}\right\rangle u_{\|}\right)= \\
=n_{i} M_{i}\left\langle\widetilde{u}_{\|} \widetilde{\mathbf{v}}_{E}\right\rangle \cdot \nabla u_{\|}-n_{i} M_{i} \nu_{d} u^{\theta} u_{\|} b^{\theta}
\end{array}
$$

for the profile, and

$$
\begin{aligned}
\frac{\partial}{\partial t}\left(n_{i} M_{i}\left\langle\frac{\widetilde{u}_{\|}^{2}}{2}\right\rangle\right)+\nabla & \cdot\left(n_{i} M_{i}\left\langle\widetilde{\mathbf{v}}_{E} \frac{\widetilde{u}_{\|}^{2}}{2}\right\rangle+M_{i}\left\langle\widetilde{n}_{i} \widetilde{\mathbf{v}}_{E}\right\rangle \frac{\widetilde{u}_{\|}^{2}}{2}\right)-\nabla_{\|}\left(\mu_{\|}\left\langle\widetilde{u}_{\|} \nabla_{\|} \widetilde{u}_{\|}\right\rangle\right)= \\
& =-n_{i} M_{i}\left\langle\widetilde{u}_{\|} \widetilde{\mathbf{v}}_{E}\right\rangle \cdot \nabla u_{\|}-\left\langle\widetilde{u}_{\|} \nabla_{\|} \widetilde{p}\right\rangle-\mu_{\|}\left\langle\left|\nabla_{\|} \widetilde{u}_{\|}\right|^{2}\right\rangle
\end{aligned}
$$

for the disturbances, in both of which Eq. (52) times the relevant factors of squared velocity (specific energy) is used. This also uses $\nabla \cdot n_{i} \mathbf{v}_{E}=0$ to treat the conventional nonlinearity, and in this and the other disturbance energy equations the resulting term in the energy flux is a third order nonlinearity which represents the ExB transport of wave energy in the turbulence. We carry these terms here for illustration, but since they are down by two orders of $\delta$ they are always small in practice (and are measured to be small in turbulence computations).

The energy transfer via the Reynolds stress is apparent in Eq. (57), as are the parallel flow transfer effects we saw in Section III. These four Eqs. $(53,54,56,57)$ together show why the particle flux has to be kept in advection; otherwise we would have problems with simultaneous conservation of energy and momentum. The same is true for the perpendicular flow, which we treat next.

The perpendicular flow is determined by the scalar fields $\phi$ and $p_{i}$, and since the ion pressure has its own equation this one becomes the equation for $\phi$. For the profiles we have

$$
\begin{aligned}
& \nabla \cdot \frac{n_{i} M_{i} c^{2}}{B^{2}} \frac{\partial}{\partial t}\left(\nabla_{\perp} \phi+\frac{\nabla_{\perp} p_{i}}{n_{e} e}\right)= \\
& \quad=\nabla \cdot \frac{c}{B^{2}} \mathbf{B} \times\left(M_{i}\left\langle\widetilde{n}_{i} \widetilde{\mathbf{v}}_{E}\right\rangle \cdot \nabla \mathbf{u}_{\perp}+\nabla \cdot n_{i} M_{i}\left\langle\widetilde{\mathbf{v}}_{E} \widetilde{\mathbf{v}}_{E}\right\rangle+n_{i} M_{i} \nu_{d} u^{\theta} \nabla \theta\right)
\end{aligned}
$$

in which the only effects are nonlinear forcing and frictional damping, as with the $u_{\|}$ profile. As in Eq. (53), we rewrite the $\widetilde{\mathbf{v}}_{E} \cdot \nabla \widetilde{\mathbf{v}}_{E}$ term as a Reynolds stress. The more 
familiar linear terms and the conventional vorticity nonlinearity are all in the equation for the disturbances,

$$
\begin{aligned}
\nabla \cdot \frac{n_{i} M_{i} c^{2}}{B^{2}} & \frac{\partial}{\partial t} \nabla_{\perp} \widetilde{\phi}= \\
=\nabla & \cdot \frac{c}{B^{2}} \mathbf{B} \times\left(n_{i} M_{i} \mathbf{v}_{E} \cdot \nabla \widetilde{\mathbf{v}}_{E}+n_{i} M_{i} \widetilde{\mathbf{v}}_{E} \cdot \nabla \widetilde{\mathbf{v}}_{E}+n_{i} M_{i} \widetilde{\mathbf{v}}_{E} \cdot \nabla \mathbf{u}_{\perp}\right) \\
& \quad+\nabla \cdot\left(\widetilde{J}_{\|} \mathbf{b}\right)+\nabla \cdot \frac{c}{B^{2}} \mathbf{B} \times \nabla \widetilde{p}
\end{aligned}
$$

neglecting the frictional damping due to its slow time scale. We are keeping only $\widetilde{\phi}$ in the vorticity disturbances, as the ExB contributions to the Reynolds stress are dominant. The first term on the right side illustrates ExB shearing but will drop out of the energy equation due to symmetry. The next term is the conventional nonlinearity and will give rise to the ExB wave energy transport. The third term is the advection of the flow profile by flow disturbances, and is the term which conserves energy against the Reynolds stress in Eq. (58).

The energy equation for the flow profile is constructed as in Section III: we multiply Eq. (58) by $-\phi$ and manipulate the divergences (equivalent to integrations by parts under an integral), also subtracting $\left(\mathbf{J}_{p} / n_{e} e\right) \cdot \nabla p_{i}$ from both sides, the left side completing the specific energy (squared velocity) and the right side providing the polarisation transfer with the ion pressure. Eq. (52) is used to bring the factors of $n_{i}$ under the partial time derivatives. For the profile we have

$$
\begin{array}{r}
\frac{\partial}{\partial t}\left(n_{i} M_{i} \frac{u_{\perp}^{2}}{2}\right)+\nabla \cdot\left(\phi \mathbf{J}_{p}+M_{i}\left\langle\widetilde{n}_{i} \widetilde{\mathbf{v}}_{E}\right\rangle \frac{u_{\perp}^{2}}{2}+n_{i} M_{i}\left\langle\widetilde{\mathbf{v}}_{E} \widetilde{\mathbf{v}}_{E}\right\rangle \cdot \mathbf{u}_{\perp}\right)= \\
=n_{i} M_{i}\left\langle\widetilde{\mathbf{v}}_{E} \widetilde{\mathbf{v}}_{E}\right\rangle: \nabla \mathbf{u}_{\perp}-n_{i} M_{i} \nu_{d} u^{\theta} u_{\perp}^{\theta}-\mathbf{J}_{p} \cdot \frac{\nabla p_{i}}{n_{e} e}
\end{array}
$$

where $u_{\perp}^{\theta}=\mathbf{u}_{\perp} \cdot \nabla \theta$, and

$$
\begin{aligned}
\mathbf{J}_{p}=-n_{i} M_{i} & \frac{\partial}{\partial t}\left(\nabla_{\perp} \phi+\frac{\nabla_{\perp} p_{i}}{n_{e} e}\right)+ \\
& +\frac{c}{B^{2}} \mathbf{B} \times\left(M_{i}\left\langle\widetilde{n}_{i} \widetilde{\mathbf{v}}_{E}\right\rangle \cdot \mathbf{u}_{\perp}+\nabla \cdot n_{i} M_{i}\left\langle\widetilde{\mathbf{v}}_{E} \widetilde{\mathbf{v}}_{E}\right\rangle+n_{i} M_{i} \nu_{d} u^{\theta} \nabla \theta\right)
\end{aligned}
$$

is the polarisation current, and for the disturbances we have

$$
\begin{aligned}
\frac{\partial}{\partial t}\left(n_{i} M_{i}\left\langle\frac{\widetilde{u}_{\perp}^{2}}{2}\right\rangle\right)+\nabla \cdot\left(M_{i}\left\langle\widetilde{n}_{i} \widetilde{\mathbf{v}}_{E}\right\rangle\left\langle\frac{\widetilde{u}_{\perp}^{2}}{2}\right\rangle+n_{i} M_{i}\left\langle\frac{\widetilde{u}_{\perp}^{2}}{2} \widetilde{\mathbf{v}}_{E}\right\rangle+\right. \\
\left.\quad+\left\langle\widetilde{\phi} \widetilde{\mathbf{J}}_{p}\right\rangle+\left\langle\widetilde{\phi} \widetilde{J}_{\|}\right\rangle+\left\langle\widetilde{\phi}_{*}\right\rangle+\left\langle\widetilde{p}_{E}\right\rangle\right) \\
=-n_{i} M_{i}\left\langle\widetilde{\mathbf{v}}_{E} \widetilde{\mathbf{v}}_{E}\right\rangle: \nabla \mathbf{u}_{\perp}+\left\langle\widetilde{J}_{\|} \nabla_{\|} \widetilde{\phi}\right\rangle-\left\langle\widetilde{p} \nabla \cdot \widetilde{\mathbf{v}}_{E}\right\rangle
\end{aligned}
$$


where $\widetilde{u}_{\perp}^{2}=\widetilde{\mathbf{v}}_{E}^{2}$ since we are keeping only ExB vorticity in the disturbances. We wee that the Reynolds stress transfers within the flow energy between profile and disturbances, and the disturbances then transfer to the pressures $\left(\widetilde{p} \nabla \cdot \widetilde{\mathbf{v}}_{E}\right)$ and the magnetic disturbances $\left(\widetilde{J}_{\|} \nabla_{\|} \widetilde{\phi}\right)$. The wave energy transport is small by $O\left(\delta^{2}\right)$ compared to either $\left\langle p \mathbf{v}_{E}\right\rangle$ or $\left\langle\phi \mathbf{J}_{\perp}\right\rangle$. The cross flux surface Alfvén wave flux $\left\langle\widetilde{\phi} \widetilde{J}_{\|} \widetilde{\mathbf{b}}_{\perp}\right\rangle$ is also small by $O\left(\delta^{2}\right)$.

The electron and ion pressure equations are somewhat easier, since they are already written in conservative form. We keep the most important quadratic fluxes, plus all the transfer effects of the disturbances. For the electrons we have

$$
\begin{aligned}
\frac{3}{2} \frac{\partial p_{e}}{\partial t}+\nabla \cdot\left(\frac{3}{2}\left\langle\widetilde{p}_{e} \widetilde{\mathbf{v}}_{E}\right\rangle+\left\langle\widetilde{q}_{e \|} \widetilde{\mathbf{b}}_{\perp}\right\rangle+\frac{5}{2} p_{e}\left\langle\widetilde{u}_{\|} \widetilde{\mathbf{b}}_{\perp}\right\rangle\right)= \\
=\left\langle\widetilde{u}_{\|} \nabla_{\|} \widetilde{p}_{e}\right\rangle-\frac{1}{n_{e} e}\left\langle\widetilde{J}_{\|} \nabla_{\|} \widetilde{p}_{e}\right\rangle-\left\langle\widetilde{p}_{e} \nabla \cdot \widetilde{\mathbf{v}}_{E}\right\rangle+\eta_{\|} J_{\|}^{2}+\left\langle\widetilde{J}_{\|} \widetilde{R}_{e i}\right\rangle
\end{aligned}
$$

neglecting the magnetic flutter transport involving $\widetilde{J}_{\|}$. For the ions we have

$$
\begin{aligned}
\frac{3}{2} \frac{\partial p_{i}}{\partial t}+\nabla \cdot\left(\frac{3}{2}\left\langle\widetilde{p}_{i} \widetilde{\mathbf{v}}_{E}\right\rangle+\left\langle\widetilde{q}_{i \|} \widetilde{\mathbf{b}}_{\perp}\right\rangle+\frac{5}{2} p_{i}\left\langle\widetilde{u}_{\|} \widetilde{\mathbf{b}}_{\perp}\right\rangle\right)= \\
=\left\langle\widetilde{u}_{\|} \nabla_{\|} \widetilde{p}_{i}\right\rangle-\left\langle\widetilde{p}_{i} \nabla \cdot \widetilde{\mathbf{v}}_{E}\right\rangle+\left\langle\widetilde{\mathbf{u}}_{p} \cdot \nabla \widetilde{p}_{i}\right\rangle+ \\
\quad+n_{i} M_{i} \nu_{d} u^{\theta} u^{\theta}+\frac{\mathbf{J}_{p}}{n_{e} e} \cdot \nabla p_{i}+\mu_{\|}\left\langle\left|\nabla_{\|} \widetilde{u}_{\|}\right|^{2}\right\rangle
\end{aligned}
$$

correspondingly neglecting the transport due to $\mathbf{u}_{p}$. This is the form of the pressure equations including magnetic flutter transport; formally the part of that due to $\widetilde{u}_{\|}$goes together with the transfers adding up to $\left\langle\widetilde{u}_{\|} \nabla_{\|} \tilde{p}\right\rangle$, but while the transfer effects remain, the transport effects are overshadowed by the contributions to $\left\langle\widetilde{p}_{E}\right\rangle$, so we drop flutter transport at this stage. Since Reynolds stress is not a proper viscosity, it does not a priori appear in the ion pressure equation. It represents a transfer effect with the disturbances, which in turn transfer the energy via other channels with the ion pressure, most notably through sound waves and through some of the electron channels, as we will see.

The magnetic field evolves through flux diffusion,

$$
\frac{1}{c} \frac{\partial A_{\|}}{\partial t}=E_{L}-\eta_{\|} J_{\|}
$$

balanced by the loop voltage $E_{L}$, here also neglecting magnetic flutter transport (always small here unless driven by reconnection, i.e., a current gradient). The energy in the disturbances evolves according to

$$
\frac{\partial}{\partial t} \frac{1}{8 \pi}\left\langle\left|\widetilde{\mathbf{B}}_{\perp}\right|^{2}\right\rangle-\nabla \cdot\left(\frac{1}{4 \pi} \frac{\partial \widetilde{A}_{\|}}{\partial t} \nabla_{\perp} \widetilde{A}_{\|}\right)=\frac{1}{n_{e} e}\left\langle\widetilde{J}_{\|} \nabla_{\|} \widetilde{p}_{e}\right\rangle-\left\langle\widetilde{J}_{\|} \nabla_{\|} \widetilde{\phi}\right\rangle-\left\langle\widetilde{J}_{\|} \widetilde{R}_{e i}\right\rangle
$$




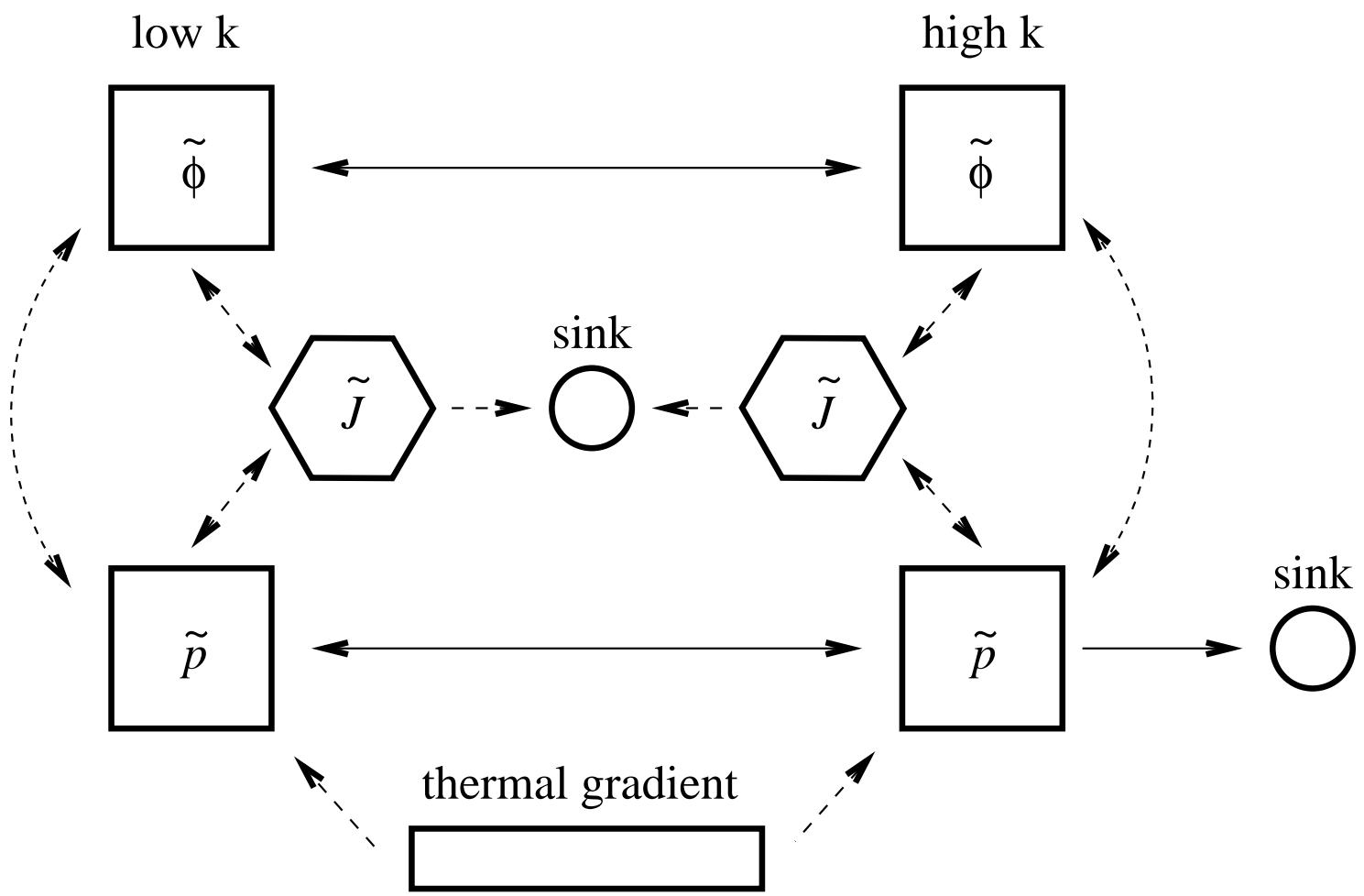

Figure 1. Energy transfer diagram for the disturbances in drift wave turbulence. Large and small scales are indentified as low and high $k_{\perp}$, respectively. The main state variables are $\widetilde{\phi}$ and $\widetilde{p}_{e}$ and $\widetilde{p}_{i}$, the latter two drawn collectively as $\widetilde{p}$. The transfer channel $\widetilde{\phi} \leftrightarrow \widetilde{J}_{\|} \leftrightarrow \widetilde{p}_{e}$ maintains drift wave mode structure, while toroidal compression $\widetilde{p} \leftrightarrow \widetilde{\phi}$ represents interchange forcing, especially important for ITG turbulence since the channel through $\widetilde{J}_{\|}$does not directly affect the ions. The weaker transfer channel $\widetilde{p}_{e} \leftrightarrow \widetilde{u}_{\|} \leftrightarrow \widetilde{p}_{i}$ representing the sound waves is not shown. Transfer between scales proceeds through the polarisation nonlinearity for $\widetilde{\phi}$ and the ExB advection nonlinearity for $\widetilde{p}$. The principal sink is diffusive mixing of $\widetilde{p}$ out of the energy producing spectral range by ExB eddies, and the resistive sink is also shown. The source is the background pressure gradient.

keeping $\widetilde{R}_{e i}$ general as it also contains parallel gradients of disturbances (cf. Eq. 32).

The key question is what to do with the equations for the disturbed energies in a transport model. A reasonable treatment is to note that over an ensemble average the partial time derivatives are zero. The energy content of the disturbances is to be neglected in any case, and since the wave energy transport terms are all small by $O\left(\delta^{2}\right)$ we drop them as well. These equations then merely become consistency relations among all the 
transfer effects. Eqs. $(57,62,66)$ respectively become

$$
\begin{gathered}
\left\langle\widetilde{u}_{\|} \nabla_{\|} \widetilde{p}_{i}\right\rangle+\mu_{\|}\left\langle\left|\nabla_{\|} \widetilde{u}_{\|}\right|^{2}\right\rangle=-n_{i} M_{i}\left\langle\widetilde{u}_{\|} \widetilde{\mathbf{v}}_{E}\right\rangle \cdot \nabla u_{\|}-\left\langle\widetilde{u}_{\|} \nabla_{\|} \widetilde{p}_{e}\right\rangle \\
\left\langle\widetilde{p}_{i} \nabla \cdot \widetilde{\mathbf{v}}_{E}\right\rangle=-n_{i} M_{i}\left\langle\widetilde{\mathbf{v}}_{E} \widetilde{\mathbf{v}}_{E}\right\rangle: \nabla \mathbf{u}_{\perp}+\left\langle\widetilde{J}_{\|} \nabla_{\|} \widetilde{\phi}\right\rangle-\left\langle\widetilde{p}_{e} \nabla \cdot \widetilde{\mathbf{v}}_{E}\right\rangle \\
\left\langle\widetilde{J}_{\|} \widetilde{R}_{e i}\right\rangle-\frac{1}{n_{e} e}\left\langle\widetilde{J}_{\|} \nabla_{\|} \widetilde{p}_{e}\right\rangle=-\left\langle\widetilde{J}_{\|} \nabla_{\|} \widetilde{\phi}\right\rangle
\end{gathered}
$$

which will be used to evaluate their the left sides in the equations for $p_{i}, p_{i}$, and $p_{e}$, respectively. In contrast to the turbulence, whose nonlinear character is sensitive to the average size of the transfer effects (i.e., the standard deviation) [7], the profiles sense only the average transfer and transport (i.e., the mean). Some of the transfer effects cancel in this fashion, most notably the adiabatic transfer mechanism $\left\langle\widetilde{J}_{\|} \nabla_{\|} \widetilde{p}_{e}\right\rangle$. The transfer dynamics in ExB turbulence is depicted in Fig. 1, with the nonlinear drift wave and interchange mechanisms highlighted. All of the arrows are bi-directional, due to the fact that the mixing and scattering tendencies in the nonlinearities, which transfer between scales of motion, impose their quasi-random character on the linear mechanisms which transfer between the state variables $\widetilde{p}_{e}, \widetilde{p}_{i}$, and $\widetilde{\phi}$, in some cases with the flux variables $\widetilde{J}_{\|}$ and $\widetilde{u}_{\|}$as intermediaries.

The transport model consists of equations for the usual three thermodynamic state variables, plus two more for the flow quantities, plus one more for the magnetic flux. It is written as follows:

$$
\begin{gathered}
\frac{\partial n_{e}}{\partial t}+\nabla \cdot \boldsymbol{\Gamma}=0 \\
\frac{3}{2} \frac{\partial p_{e}}{\partial t}+\nabla \cdot \mathbf{Q}_{e}=\mathbf{T}_{e i}+\eta_{\|} J_{\|}^{2} \\
\frac{3}{2} \frac{\partial p_{i}}{\partial t}+\nabla \cdot \mathbf{Q}_{i}=-\mathbf{T}_{e i}-\mathbf{R}_{E}: \nabla \mathbf{u}_{\perp}-\mathbf{R}_{E \|} \cdot \nabla u_{\|}+n_{i} M_{i} \nu_{d} u^{\theta} u^{\theta}+\frac{\mathbf{J}_{p}}{n_{e} e} \cdot \nabla p_{i} \\
\nabla \cdot \frac{n_{i} M_{i} c^{2}}{B^{2}} \frac{\partial}{\partial t}\left(\nabla_{\perp} \phi+\frac{\nabla_{\perp} p_{i}}{n_{e} e}\right)=\nabla \cdot \frac{c}{B^{2}} \mathbf{B} \times\left(M_{i} \boldsymbol{\Gamma} \cdot \nabla \mathbf{u}_{\perp}+\nabla \cdot \mathbf{R}_{E}+n_{i} M_{i} \nu_{d} u^{\theta} \nabla \theta\right) \\
n_{i} M_{i} \frac{\partial u_{\|}}{\partial t}+M_{i} \boldsymbol{\Gamma} \cdot \nabla u_{\|}+\nabla \cdot \mathbf{R}_{E \|}+n_{i} M_{i} \nu_{d} u^{\theta} b^{\theta}=0 \\
\frac{1}{c} \frac{\partial A_{\|}}{\partial t}=E_{L}-\eta_{\|} J_{\|}
\end{gathered}
$$

The parallel current is given by Ampere's law,

$$
J_{\|}=-\frac{c}{4 \pi} \nabla_{\perp}^{2} A_{\|}
$$


The following transport quantities are defined as ensemble averages over the turbulence:

$$
\begin{gathered}
\boldsymbol{\Gamma}=\left\langle\widetilde{n}_{e} \widetilde{\mathbf{v}}_{E}\right\rangle \quad \mathbf{Q}_{e}=\frac{3}{2}\left\langle\widetilde{p}_{e} \widetilde{\mathbf{v}}_{E}\right\rangle \quad \mathbf{Q}_{i}=\frac{3}{2}\left\langle\widetilde{p}_{i} \widetilde{\mathbf{v}}_{E}\right\rangle \\
\mathbf{R}_{E}=n_{i} M_{i}\left\langle\widetilde{\mathbf{v}}_{E} \widetilde{\mathbf{v}}_{E}\right\rangle \\
\mathbf{R}_{E \|}=n_{i} M_{i}\left\langle\widetilde{u}_{\|} \widetilde{\mathbf{v}}_{E}\right\rangle \\
\mathbf{T}_{e i}=\left\langle\widetilde{u}_{\|} \nabla_{\|} \widetilde{p}_{e}\right\rangle-\left\langle\widetilde{p}_{e} \nabla \cdot \widetilde{\mathbf{v}}_{E}\right\rangle-\left\langle\widetilde{J}_{\|} \nabla_{\|} \widetilde{\phi}\right\rangle
\end{gathered}
$$

giving the particle flux, electron and ion heat fluxes, the perp-perp and perp-parallel Reynolds stresses, and the anomalous heat transfer, respectively.

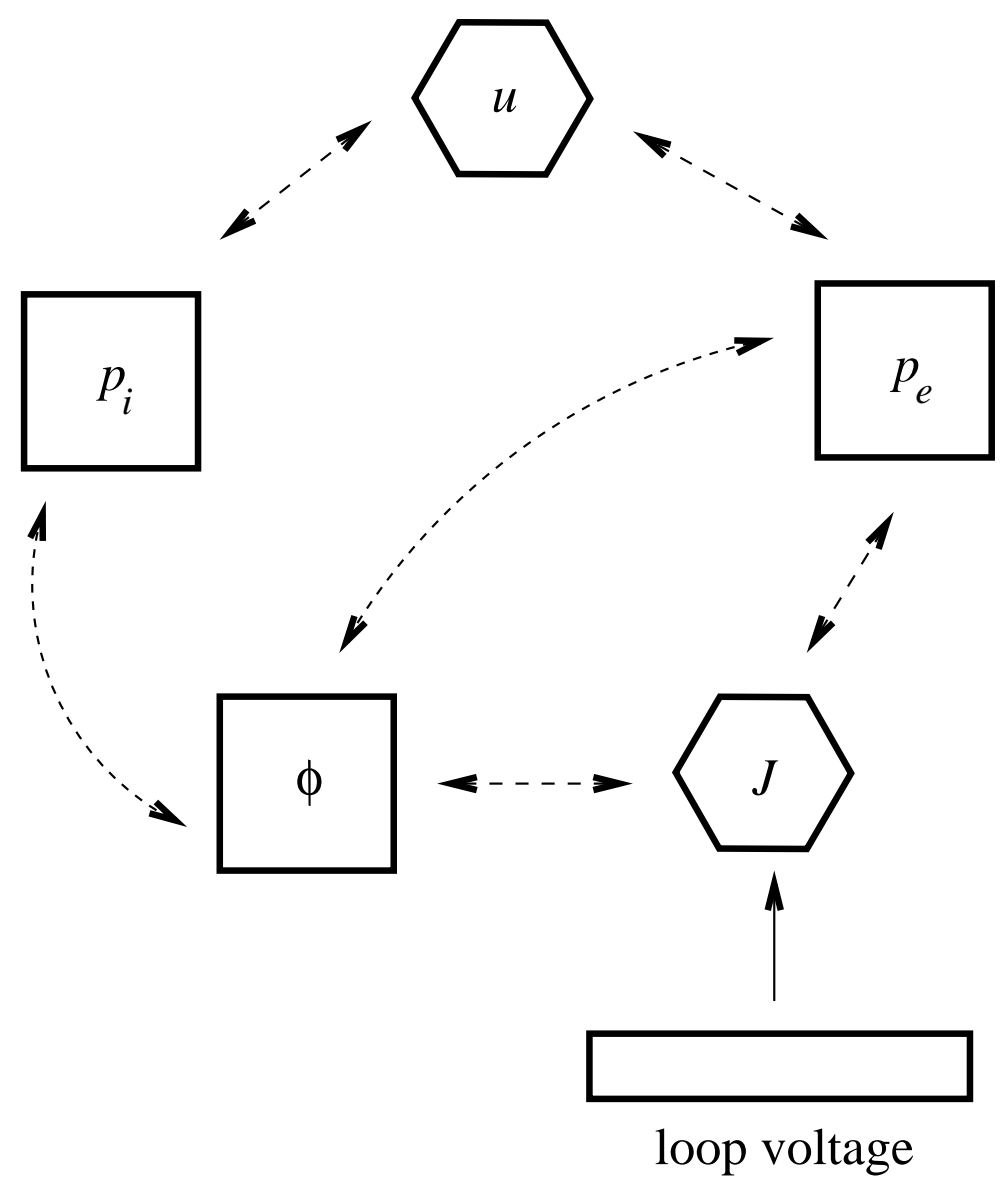

Figure 2. Energy transfer diagram for the profile quantities in a transport model. The five quantities representing energy in Eqs. (71-74) are shown, with the transfer channels representing the disturbances. The three pathways connecting $p_{e}$ to $p_{i}$ represent the three terms in $\mathbf{T}_{e i}$ in Eq. (79). The source is the loop voltage. The only sink is transport through the outer boundary. 
The perpendicular flow and polarisation current are given by

$$
\begin{gathered}
\mathbf{u}_{\perp}=\frac{c}{B^{2}} \mathbf{B} \times\left(\nabla \phi+\frac{\nabla p_{i}}{n_{e} e}\right) \\
\mathbf{J}_{p}=\frac{c}{B^{2}} \mathbf{B} \times\left(n_{i} M_{i} \frac{\partial \mathbf{u}_{\perp}}{\partial t}+M_{i} \boldsymbol{\Gamma} \cdot \nabla \mathbf{u}_{\perp}+\nabla \cdot \mathbf{R}_{E}+n_{i} M_{i} \nu_{d} u^{\theta} \nabla \theta\right)
\end{gathered}
$$

and poloidal contravariant components are denoted by the superscript $\theta$. As explicit dissipation mechanisms we have the neoclassical friction $\nu_{d}$ and the parallel resistivity $\eta_{\|}$. The only source in the model as written is the loop voltage $E_{L}$.

The most important result of this model is the set of transfer channels between each of the pressures and the perpendicular and parallel flows, which are depicted in Fig. 2. This includes the appearance of anomalous transfer effects between the electron and ion temperatures, given by $\mathbf{T}_{e i}$ as defined in Eq. (79). This anomalous transfer is an essential result of drift wave physics in the turbulence. The electron thermal gradient drives ExB motion through the adiabatic and Alfvénic couplings, $\widetilde{p}_{e} \leftrightarrow \widetilde{J}_{\|} \leftrightarrow \widetilde{\phi}$. Dissipation takes place in both the electron $\left(\widetilde{J}_{\|}\right)$and ion $\left(\widetilde{u}_{\|}\right)$channels, which is here represented in terms of the coupling between the pressures. More generally, whichever species has the larger temperature contributes more to the drive of the turbulence, relaxing the corresponding gradient the more efficiently. Rather than a monotonic exchange effect as would be mandated by dissipative kinetic theory, the anomalous transfer is more a tendency towards equipartition given a statistical system. All three of the terms which comprise $\mathbf{T}_{e i}$ in Eq. (79) are involved, but $\left\langle\widetilde{J}_{\|} \nabla_{\|} \widetilde{\phi}\right\rangle$ is the strongest for drift wave turbulence, $\left\langle\widetilde{p}_{e} \nabla \cdot \widetilde{\mathbf{v}}_{E}\right\rangle$ is strongest for interchange turbulence, and $\left\langle\widetilde{u}_{\|} \nabla_{\|} \widetilde{p}_{e}\right\rangle$ is strongest for ITG turbulence (the pure toroidal ITG transfer goes through $\left\langle\widetilde{p}_{i} \nabla \cdot \widetilde{\mathbf{v}}_{E}\right\rangle$, i.e., directly from ion flows to the ion pressure, not between species). For strictly cold ions in the absence of flows, the balances among the disturbances (Eqs. 67-69) would indicate that $\left\langle\widetilde{J}_{\|} \nabla_{\|} \widetilde{\phi}\right\rangle$ would balance $\left\langle\widetilde{p}_{e} \nabla \cdot \widetilde{\mathbf{v}}_{E}\right\rangle$ and that $\left\langle\widetilde{u}_{\|} \nabla_{\|} \widetilde{p}_{e}\right\rangle$ would vanish and hence that $\mathbf{T}_{e i}$ would be zero on average. However, the presence of both flows as reserviors allows a finite $\mathbf{T}_{e i}$ to exist even for $T_{i}=0$. Although this probably depends in detail on the existence of direct dissipation in the ions (i.e., viscosity), and therefore a finite $T_{i}$, it is nevertheless important to the physics that these transfer channels exist and that they can be significantly stronger that the classical, collisional transfer usually considered. It is important to note that the drift wave anomalous heat transfer, $\left\langle\widetilde{J}_{\|} \nabla_{\|} \widetilde{\phi}\right\rangle$, representing compression of the polarisation drift in the ions and parallel currents in the electrons, can be significant even for $T_{i}=0$.

The other significant transfer effects are those involving the Reynolds stresses. The one most considered is $\mathbf{R}_{E}$, acting on the perpendicular flow. But in regions where the 
parallel flow has a large gradient the perp-parallel component $\mathbf{R}_{E \|}$ also enters. It is interesting to note that the direct dissipation in this model due to $\nu_{d}$ and $\mathbf{R}_{E}$ acting on the diamagnetic flow $\mathbf{u}_{*}$ is exactly cancelled by part of the polarisation transfer, leaving only the time dependent and advective vorticity contributions, which constitute a real compressional effect. This does not reduce the computational complexity however. But since Eq. (73) says that $\nabla \cdot \mathbf{J}_{p}=0$, the polarisation current $\mathbf{J}_{p}$ may itself vanish in an enclosed domain, allowing the neglect of the work term involving $\mathbf{J}_{p}$ entirely. This only holds if the gradients of $\phi$ and $p_{i}$ are set to zero at the outer boundary, however; if it is desired to incorporate a loss model at that boundary, then one must keep $\mathbf{J}_{p} \cdot \nabla p_{i}$ to conserve energy. The other unusual effects regarding the polarisation drift velocity are avoided because of the neglect of parallel transport in a one-dimensional model, but in a two-dimensional model they would have to be faced (see the comments preceding Eq. 36).

It might be tempting to model $\mathbf{R}_{E}$ strictly as flow generation, expecting it to balance the neoclassical friction in determining the equilibrium state of $\phi$ [18]. But in many cases the only important effect on $\phi$ is $\mathbf{R}_{E}$ acting alone; there is evidence from experimental studies of internal transport barriers that in some regimes, especially coinjection of neutral beams, (the rotation or V-cross-B terms in the force balance are stronger than the pressure gradient and therefore) the Reynolds stress balances itself to zero in response to strong flows [19], and computations of drift wave turbulence in the presence of a background ExB vorticity show that the longer wavelengths are actually driven while the shorter ones are suppressed, so that very strong flow shear would provide a net drive for the turbulence and therefore self-deplete [20]. Such a self-regulator mechanism is probably best for modelling $\mathbf{R}_{E}$. Parallel flow shear is much less well studied, so the question of what to do for $\mathbf{R}_{E \|}$ is more open.

Current transport codes neglect the partial time derivative in Eq. (73) and with it the difficulties in treating the polarisation transfer; the frictional damping then (or $\mathbf{R}_{E}$ itself) takes over the determination of $\phi$ and the energy in $\mathbf{u}_{\perp}$ is neglected. But in general $\mathbf{u}_{\perp}$ can be as large as $u_{\|}$, so it is better to keep both flow energies. Actually, most transport codes neglect the question of plasma rotation entirely, as well as the evolution of the magnetic flux, which reduces the set of equations to Eqs. (70-72) for $n_{e}, p_{e}$, and $p_{i}$, solely with the particle and energy fluxes, which are easy to model consistently. Even in that case, we do still have the anomalous transfer terms due to the disturbances, and these could become interesting in several regions of the plasma.

The existence of anomalous heat transfer has been noted before, in the context of a quasilinear model for the disturbances [21]. The more general version presented here 
in the context of mean field theory incorporates models based upon linear instabilities as a subset, but it also allows incorporation of the results from general turbulence studies, including the strong mode structure changes brought about by the nonlinear dynamics of the ExB vorticity [7]. The quasilinear model indicates that $\mathbf{T}_{e i}$ is only important where $\Gamma$ is significant; in turbulence however, especially electromagnetic turbulence, the robust activity in the dynamics involving $\nabla_{\|} \widetilde{J}_{\|}$ensures a prominent role for all three of the contributors to $\mathbf{T}_{e i}$ in Eq. (79). Ultimately, further investigation and diagnosis of generalised turbulence computations will be required to validate any strong conclusions involving these effects.

\section{The Characteristics of Transport Caused by ExB Turbulence}

In this section we confirm the main points of Section II concerning the special nature of ExB turbulent transport, compared with the more familiar random scattering via Coulomb collisions.

The first point is the simplest: the ExB velocity is nearly divergence free,

$$
\nabla \cdot \mathbf{v}_{E} \approx 0
$$

and in a homogeneous magnetic field it is divergence free. For some laboratory configurations, cylindrical plasma geometry at very low beta bounded by plates at the ends, for example, this can be taken to be exact. The result is something well known from neutral fluid dynamics: in a divergence free velocity field the pressure does not do work on the fluid elements. The transfer of energy between the pressure and the velocity becomes small. For interchange turbulence the work done by the pressure on the ExB velocity divergence is responsible for the principal forcing effect on the velocity. But for the profiles this is overshadowed by the other transfer effects. A divergence free transporting velocity is something very different from the model in collisional kinetics, by which the velocity is given by diffusion,

$$
n_{e} \mathbf{v}_{D}=-D\left(n_{e}, T_{e}\right) \nabla n_{e}
$$

for example. This velocity is only divergence free for specific dependences of $D$ on the parameters. For example, the scale of the velocity divergence for a constant diffusion is the same as the scale of the density profile. But $\mathbf{v}_{E}$ is exactly or nearly divergence free according to the magnetic geometry, regardless of what form $n_{e}(r)$ or $T_{e}(r)$ take.

The second point is the Poynting cancellation, Eq. (42). The presence of $p \mathbf{v}_{E}$ in the transport with the factor of $3 / 2$ is true for total energy, but what we want to know is if 
that is just a result of formal manipulation. We recall that an ideal pressure equation may be written in terms of a transport term $[(5 / 2) p \mathbf{v}]$ and a work term $[\mathbf{v} \cdot \nabla p]$, or alternatively in terms of a transport term $[(3 / 2) p \mathbf{v}]$ and a divergence $[p \nabla \cdot \mathbf{v}]$. If the divergence is small, as we showed for $\mathbf{v}_{E}$, then the second form is more germane. But it is even more interesting to know how the transport works for each piece of the energy rather than the total. The quantity $\left(\phi \mathbf{J}_{\perp}+p \mathbf{v}_{E}\right)$ appears in the transport equation for the drift energy, Eq. 45. In modelling transport terms we usually write down the lowest order forms, which still conserve energy properly. We know that the diamagnetic current is at the same order as $\mathbf{v}_{E}$ if $n_{e} e \mathbf{E}_{\perp}$ is comparable to $\nabla p$, so $\phi \mathbf{J}_{\perp}$ is generally the same size as $p \mathbf{v}_{E}$. In fact they are close enough for the remnant to be negligible. A particularly useful form of the Poynting cancellation turns out to be the one with the diamagnetic current,

$$
\nabla \cdot\left(\phi \mathbf{J}_{*}+p \mathbf{v}_{E}\right)=\nabla \cdot\left[\frac{c}{B^{2}} \mathbf{B} \times \nabla(\phi p)\right]
$$

This is a magnetic divergence, and it is now down to the next smallest row of terms in Eq. (50) as its scale is that of the magnetic field ( $R$ in toroidal geometry), and the scale of interest is $L_{p}$. It is therefore a fundamental property of ExB flow dynamics that its contribution to the total energy transport is

$$
\mathbf{Q}_{e}+\mathbf{Q}_{i}=\frac{3}{2}\left\langle\widetilde{p} \widetilde{\mathbf{v}}_{E}\right\rangle
$$

given that there are no radial equilibrium flows. The physical point of this is that the energy due to the fluid perpendicular velocities presents a transfer term of the form $p \nabla \cdot \mathbf{v}_{E}$, rather than the more usual form $\mathbf{v}_{E} \cdot \nabla p$, to the thermal energy reservoir, due to the Poynting cancellation, so that the actual transport is due to the remaining $(3 / 2) p \mathbf{v}_{E}$. This role of the Poynting cancellation is what makes the ExB velocity somewhat "special" compared to all the others.

The third point concerns magnetic flux diffusion. It is an interesting measured property of magnetically confined plasma experiments that the electron thermal transport is anomalous by more than two orders of magnitude, but the magnetic flux diffusion is neoclassical [22] (classical diffusion, modified by toroidal drifts of particles whose parallel motion is in the long mean free path regime). If we write the mean field Ohm's law keeping magnetic flutter, however,

$$
\frac{1}{c} \frac{\partial A_{\|}}{\partial t}=\frac{1}{n_{e} e} \nabla \cdot\left\langle\widetilde{p}_{e} \widetilde{\mathbf{b}}_{\perp}\right\rangle-\nabla \cdot\left\langle\widetilde{\phi} \widetilde{\mathbf{b}}_{\perp}\right\rangle+E_{L}-\eta_{\|} J_{\|}
$$


we instantly see why this should be true for ExB turbulence, If the magnetic disturbances are small or, as is more often the case, uncorrelated, then the anomalous flux diffusion is small; indeed it is zero for purely electrostatic turbulence. It is well known that $\left\langle\widetilde{q}_{e \|} \widetilde{\mathbf{b}}_{\perp}\right\rangle$ contributes negligibly to transport for drift wave turbulence in either slab or toroidal geometry, due to self consistency effects between $\widetilde{\phi}$ and $\widetilde{T}_{e}[6,23]$. Only when the current gradient is available as an energy source, as for tearing instabilities, [24], do these processes lead to appreciable transport. It then follows that the anomalous flux diffusion should be small. With $J_{\|}$determined by $A_{\|}$, this holds as well for the current diffusion. Anomalous current diffusion can only result from the ExB nonlinearity in the electron inertia, and this is small compared to the mechanisms by which currents in a specifically current carrying plasma are generated. This is another nice contrast to classical diffusion, for in that case the flux diffusivity is much greater than the particle diffusivity. Neglecting $\widetilde{\mathbf{b}}_{\perp}$, the mean field Ohm's law is purely diffusive, as we can see from

$$
\frac{\partial A_{\|}}{\partial t}=E_{L}+\frac{\eta_{\|} c^{2}}{4 \pi} \nabla_{\perp}^{2} A_{\|}
$$

having inserted Eq. (76) to make the flux diffusion explicit. Comparing the classical diffusivities (assuming a classical $\eta_{\|}$), we find

$$
\frac{\eta_{\|} c^{2}}{4 \pi}=\frac{0.51}{\beta_{e}} \rho_{e}^{2} \nu_{e}=\frac{0.51}{\beta_{e}} D_{e}
$$

With $\beta_{e} \sim 10^{-4}$ in the edge regions of fusion plasmas, this classical diffusivity is as large as the anomalous electron thermal diffusivity if not larger. An interesting consequence is that the MHD equilibrium in this regime is resistive. If the pressure gradient changes during a transport event it is not the case that the magnetic structure evolves isentropically through its successive equilibria. This point has been largely ignored in edge transport modelling, which takes the magnetic structure to be fixed.

The fourth point is the various mechanisms of ExB flow generation. Self consistent flow generation by turbulence goes through the Reynolds stress [18], a fluid dynamical mechanism. But for closed, toroidal geometry a sheared ExB velocity profile also means a radial electric field with radial dependence. In other words, a finite divergence and hence a finite charge density. But within low frequency fluid drift motion at scales larger than the Debye length, the charge density is always small, specifically, much less than $n_{e} e$, the charge density of the electrons. Not only that, it is small in all phases of the dynamics, 
which we can quickly show. Writing simple continuity equations for both fluids using the velocities in Eqs. $(19,20)$, we find a charge continuity equation given by

$$
\frac{\partial \rho_{c h}}{\partial t}+\nabla \cdot\left(\rho_{c h} \mathbf{v}_{E}\right)+\nabla \cdot\left(J_{\|} \mathbf{b}+\mathbf{J}_{*}+\mathbf{J}_{p}\right)=0
$$

where $\rho_{c h}=n_{e} e-n_{i} Z e$ is the charge density. Taking into account that $\mathbf{v}_{E} \cdot \nabla \sim \partial / \partial t$, the first two terms are the same size. But the polarisation current can be written next to them,

$$
-\frac{\partial \rho_{c h}}{\partial t}-\nabla \cdot\left(\rho_{c h} \mathbf{v}_{E}\right)-\nabla \cdot \mathbf{J}_{p}=\nabla \cdot\left(J_{\|} \mathbf{b}+\mathbf{J}_{*}\right)
$$

The polarisation divergence and the charge density term have the same form, and the polarisation divergence is larger by a factor of $c^{2} / v_{A}^{2}$, since $\nabla_{\perp}^{2} \phi=4 \pi \rho_{c h}$. All of the terms involving $\rho_{c h}$ are then negligible, and we are left with $\nabla \cdot \mathbf{J}=0$ as before. This underscores the strictness of the quasineutral character of the dynamics. It holds pointwise, not just in an averaged sense. We then conclude that any mechanism which should build up an electric field does so under the ExB fluid dynamics and not due to transport of charges by currents. The total charge transport itself must vanish. This leads to what happens as a result of any charged particle source or sink mechanisms. It is quite possible to have source terms on the right side of Eq. (90), but instead of accumulating charge we then have a balance between sources and transport,

$$
-\nabla \cdot \mathbf{J}_{p}=\nabla \cdot\left(J_{\|} \mathbf{b}+\mathbf{J}_{*}\right)+S_{e}-S_{i}
$$

so that the charge content remains small. This is the high throughput regime for electric charge, similar to the situation with free energy in drift wave turbulence where the current transfers thermal and drift free energy back and forth but the magnetic energy always remains small. In the high throughput regime for charges, quasineutrality is maintained and these source mechanisms act as a torque on the ExB vorticity [12]. Taking charges out of a flux tube causes the flux tube to rotate, since while the small but finite $\nabla_{\perp}^{2} \phi$ leads to no appreciable charge density, it the ExB vorticity it does lead to has a significant role. Ultimately, the source of this angular momentum, as in any case for perpendicular momentum in fluid drift motion, is the background magnetic field.

The last point concerns anomalous momentum transport. With the ExB vorticity proportional to the small but nonzero charge density, transport of angular momentum takes place through the Reynolds stress, which is nothing more than the nonlinear polarisation current. But in modelling the transport of this current one should not use an anomalous conductivity in a simple radial Ohm's law. Conductivity, or better its inverse, resistivity, 
is a friction between the two fluids which conserves total momentum. Resistivity leads to particle diffusion, as the magnetic Lorentz force balancing resistive friction,

$$
\frac{\mathbf{v} \times \mathbf{B}}{c}=\eta_{\perp} \mathbf{J}
$$

with perpendicular resistivity $\eta_{\perp}=m_{e} \nu_{e} / n_{e} e^{2}$, gives rise to a collisional drift velocity which acts as a diffusion, once the MHD equilibrium constraint, $\mathbf{J} \times \mathbf{B}=c \nabla p$, is set in,

$$
\mathbf{v}_{D}=-\frac{D_{e}}{T_{e}} \nabla_{\perp} p
$$

(in the neglect of thermal forces). But in the momentum equation, the resistive friction cancels, as

$$
\begin{gathered}
\frac{\partial}{\partial t} n_{e} m_{e} \mathbf{v}+\nabla \cdot(\cdots)=\mathbf{R}_{i e}-\nabla p_{e}-n_{e} e\left(\mathbf{E}+\frac{\mathbf{v}}{c} \times \mathbf{B}\right) \\
\frac{\partial}{\partial t} n_{i} M_{i} \mathbf{u}+\nabla \cdot(\cdots)=-\mathbf{R}_{i e}-\nabla p_{i}+n_{i} Z e\left(\mathbf{E}+\frac{\mathbf{u}}{c} \times \mathbf{B}\right)
\end{gathered}
$$

add to become approximately

$$
\frac{\partial}{\partial t} \rho \mathbf{u}+\nabla \cdot(\cdots)=-\nabla p+\frac{\mathbf{J} \times \mathbf{B}}{c}
$$

The only phenomenon left to transport momentum is the Reynolds stress (cf. Eq. 58), which is the dominant effect in the momentum flux terms we did not explicitly write in Eqs. $(94,95)$. The JxB force remains as a transfer mechanism between fluid and Poynting momentum, with the background magnetic field acting as an anchor.

To summarise, transport of the background profile quantities by small scale ExB turbulence can still be diffusive, provided the scale of motion is small compared to $L_{p}$. But its fundamental properties are qualitatively different from those of a kinetic diffusion via random thermal motions, and these should be taken into account when constructing transport models. An example model for one-dimensional transport including flows and magnetic induction has been given in Section IV. Attempts to extend conventional twoand three-dimensional edge transport models, which are able to treat the parallel fluxes as well as the radial transport since the profiles are no longer flux functions in their regime, to incorporate these effects are ongoing [25]. 


\section{References}

[1] J. Hugill, Nucl. Fusion 23 (1983) 331.

[2] A. J. Wootton, B. A. Carreras, H. Matsumoto, K. McGuire, W. A. Peebles, Ch. P. Ritz, P. W. Terry, and S. J. Zweben, Phys. Fluids B 2 (1990) 2879.

[3] S. I. Braginskii, Rev. Plasma Phys. 1 (1965) 205.

[4] One dimensional semi-empirical transport models up to 1990 are reviewed by W. A. Houlberg, D. W. Ross, G. Bateman, S. C. Cowley, P. C. Efthimion, W. W. Pfeiffer, G. D. Porter, D. E. Shumaker, L. E. Sugiyama, and J. C. Wiley, Phys. Fluids B 2 (1990) 2913; see also [1] for the earliest efforts. Some more recent efforts are G. Becker, Nucl. Fusion 36 (1996) 1751; G. Bateman, A. Kritz, J. Kinsey, A. Redd, and J. Weiland, Phys. Plasmas 5 (1998) 1793; P. Strand, H. Nordman, J. Weiland, and J. Christiansen, Nucl. Fusion 38 (1998) 545; G. Vlad, M. Marinucci, F. Romanelli, A. Cherubini, M. Erba, V. Parail, and A. Taroni, Nucl. Fusion 38 (1998) 557. Two dimensional edge transport models start with the B2 code, B. Braams, NET Report No. 68, January 1987 (EUR-FU/XII-80/87/68), A Multi-Fluid Code for Simulation of the Edge Plasma in Tokamaks; R. Schneider, B. Braams, D. Reiter, H. Zehrfeld, J. Neuhauser, M. Baelmans, H. Kastelewicz, and R. Wunderlich, Contrib. Plasma Phys. 32 (1992) 450; and the UEDGE code, T. Rognlien, P. Brown, T. Campbell et al, Contrib. Plasma Phys. 34 (1994) 362; T. Rognlien, J. Milovich, M. Resnick, and G. Porter, J. Nucl. Mater. 196198 (1002) 347.

[5] A. H. Boozer, Phys. Fluids B 4 (1992) 2845.

[6] B. Scott, Plasma Phys. Contr. Fusion 39 (1997) 1635.

[7] B. Scott, Phys. Plasmas 7 (2000) 1845.

[8] A. Hasegawa and K. Mima, Phys. Rev. Lett. 39 (1977) 205; Phys. Fluids 21 (1978) 87.

[9] M. Wakatani and A. Hasegawa, Phys. Fluids 27 (1984) 611.

[10] E. A. Frieman and Liu Chen, Phys. Fluids 25 (1982) 502.

[11] T. E. Stringer, Plasma Phys. Contr. Fusion 33 (1991) 1715; D. W. Ross, Plasma Phys. Contr. Fusion 34 (1992) 137. 
[12] K Lackner, private discussions, 1996.

[13] F. L. Hinton and C. W. Horton, Jr, Phys. Fluids 14 (1971) 116.

[14] H. Strauss, Phys. Fluids 19 (1976) 134.

[15] J. F. Drake and T. M. Antonsen, Jr., Phys. Fluids 27 (1984) 898.

[16] A. Smolyakov, Canadian J. Phys. 76 (1998) 321.

[17] S.-T. Tsai, F. W. Perkins, and T. H. Stix, Phys. Fluids 13 (1970) 2108.

[18] P. Diamond and Y. Kim, Phys. Fluids B 3 (1991) 1626; for a recent review see also P. Terry, Rev. Mod. Phys. 72 (2000) 109.

[19] E. J. Synakowski, S. H. Batha, M. A. Beer, M. G. Bell, R. E. Bell, R. V. Budny, C. E. Bush, P. C. Efthimion, T. S. Hahm, G. W. Hammett, B. LeBlanc, F. Levinton, E. Mazzucato, H. Park, A. T. Ramsey, G. Schmidt, G. Rewoldt, S. D. Scott, G. Taylor, and M. C. Zarnstorff, Phys. Plasmas 4 (1997) 1736.

[20] B Scott, Plasma Phys. Contr. Fusion 34 (1992) 1977.

[21] R. E. Waltz, G. M. Staebler, W. Dorland, G. W. Hammett, M. Kotschenreuther, and J. A. Konings, Phys. Plasmas 4 (1997) 2482.

[22] M. C. Zarnstorff, K. McGuire, M. G. Bell, B. Grek, D. Johnson, D. McCune, H. Park, A. Ramsey, and G. Taylor, Phys. Fluids B 2 (1990) 1852.

[23] R. E. Waltz, Phys. Fluids 28 (1985) 577.

[24] H. Furth, J. Killeen, and M. Rosenbluth, Phys. Fluids 6 (1963) 453.

[25] T. Rognlien, G. Porter, and D. Ryutov, J. Nucl. Mater. $266 \& 269$ (1999) 654; D. Morozov, V. Rozhansky, J. Herrera, and T. Soboleva, Phys. Plasmas 7 (2000) 1184. 